\title{
PREDICTING FUTURE SPACE NEAR-IR GRISM SURVEYS USING THE WFC3 INFRARED SPECTROSCOPIC PARALLELS SURVEY
}

\author{
James W. Colbert ${ }^{1}$, Harry Teplitz ${ }^{2}$, Hakim AteK ${ }^{1,3}$, Andrew Bunker ${ }^{4}$, Marc Rafelski $^{2}$, Nathaniel Ross $^{5}$, \\ Claudia Scarlata $^{6}$, Alejandro G. Bedregal ${ }^{6,7}$, Alberto Dominguez $^{8}$, Alan Dressler $^{9}$, Alaina Henry $^{10}$, \\ Matt Malkan $^{5}$, Crystal L. Martin ${ }^{11}$, Dan Masters ${ }^{8,9}$, Patrick McCarthy $^{9}$, And Brian Siana ${ }^{8}$ \\ ${ }^{1}$ Spitzer Science Center, California Institute of Technology, Pasadena, CA 91125, USA \\ ${ }^{2}$ Infrared Processing and Analysis Center, Caltech, Pasadena, CA 91125, USA \\ ${ }^{3}$ Laboratoire d'astrophysique, École Polytechnique Fédérale de Lausanne, Observatoire de Sauverny, 1290 Versoix, Switzerland \\ ${ }^{4}$ Department of Physics, University of Oxford, Denys Wilkinson Building, Keble Road, Oxford OX1 3RH, UK \\ ${ }^{5}$ Department of Physics and Astronomy, University of California, Los Angeles, CA, USA \\ ${ }^{6}$ Minnesota Institute for Astrophysics, University of Minnesota, Minneapolis, MN 55455, USA \\ ${ }^{7}$ Department of Physics and Astronomy, Tufts University, Medford, MA 02155, USA \\ 8 Department of Physics and Astronomy, University of California Riverside, Riverside, CA 92521, USA \\ ${ }^{9}$ Observatories of the Carnegie Institution for Science, Pasadena, CA 91101, USA \\ ${ }^{10}$ Astrophysics Science Division, Goddard Space Flight Center, Code 665, Greenbelt, MD 20771, USA \\ ${ }^{11}$ Department of Physics, University of California, Santa Barbara, CA 93106, USA \\ Received 2013 May 6; accepted 2013 October 19; published 2013 November 22
}

\begin{abstract}
We present near-infrared emission line counts and luminosity functions from the Hubble Space Telescope Wide Field Camera 3 Infrared Spectroscopic Parallels (WISP) program for 29 fields $\left(0.037 \mathrm{deg}^{2}\right)$ observed using both the G102 and G141 grism. Altogether we identify 1048 emission line galaxies with observed equivalent widths greater than $40 \AA, 467$ of which have multiple detected emission lines. We use simulations to correct for significant (>20\%) incompleteness introduced in part by the non-dithered, non-rotated nature of the grism parallels. The WISP survey is sensitive to fainter flux levels $\left((3-5) \times 10^{-17} \mathrm{erg} \mathrm{s}^{-1} \mathrm{~cm}^{-2}\right)$ than the future space near-infrared grism missions aimed at baryonic acoustic oscillation cosmology $\left((1-4) \times 10^{-16} \mathrm{erg} \mathrm{s}^{-1} \mathrm{~cm}^{-2}\right)$, allowing us to probe the fainter emission line galaxies that the shallower future surveys may miss. Cumulative number counts of $0.7<z<1.5$ galaxies reach 10,000 $\mathrm{deg}^{-2}$ above an $\mathrm{H} \alpha$ flux of $2 \times 10^{-16} \mathrm{erg} \mathrm{s}^{-1} \mathrm{~cm}^{-2}$. H $\alpha$-emitting galaxies with comparable [O III] flux are roughly five times less common than galaxies with just $\mathrm{H} \alpha$ emission at those flux levels. Galaxies with low $\mathrm{H} \alpha /[\mathrm{O} \mathrm{III}]$ ratios are very rare at the brighter fluxes that future near-infrared grism surveys will probe; our survey finds no galaxies with $\mathrm{H} \alpha /[\mathrm{O} \mathrm{III}]<0.95$ that have $\mathrm{H} \alpha$ flux greater than $3 \times 10^{-16} \mathrm{erg} \mathrm{s}^{-1} \mathrm{~cm}^{-2}$. Our $\mathrm{H} \alpha$ luminosity function contains a comparable number density of faint line emitters to that found by the Near IR Camera and Multi-Object Spectrometer near-infrared grism surveys, but significantly fewer (factors of 3-4 less) high-luminosity emitters. We also find that our high-redshift $(z=0.9-1.5)$ counts are in agreement with the high-redshift $(z=1.47)$ narrowband $\mathrm{H} \alpha$ survey of HiZELS (Sobral et al.), while our lower redshift luminosity function $(z=0.3-0.9)$ falls slightly below their $z=0.84$ result. The evolution in both the H $\alpha$ luminosity function from $z=0.3-1.5$ and the [O III] luminosity function from $z=0.7-2.3$ is almost entirely in the $L_{\star}$ parameter, which steadily increases with redshift over those ranges.
\end{abstract}

Key words: galaxies: evolution - galaxies: high-redshift - galaxies: luminosity function, mass function

Online-only material: color figures

\section{INTRODUCTION}

The majority of star formation, supermassive black hole accretion, and galaxy assembly in the universe likely occurred over the epoch of $z=0.7-2$ (e.g., McLure et al. 2006; PérezGonzález et al. 2008; van Dokkum et al. 2010; Magnelli et al. 2011; Muzzin et al. 2013; Ilbert et al. 2013). A careful census of the spatial distribution of galaxies in this redshift range is critical for measuring the large-scale clustering of galaxies that results from baryonic acoustic oscillations (BAO), which will enable us to probe the expansion history of the universe and address the equation state of dark energy (Eisenstein \& Hu 1998; Cole et al. 2005; Eisenstein et al. 2005; Weinberg et al. 2012). Large near-infrared spectroscopic surveys are required to study this redshift regime, as the most luminous nebular emission lines move out of the optical at these redshifts.

Ground-based searches for emission lines from faint highredshift galaxies are severely impacted by the bright near-IR airglow, which effectively eliminates the possibility of slitless grism spectroscopy. Most near-infrared ground spectroscopy is done a single object at a time, requiring some form of preselection that generally biases against discovery of the highest equivalent width (EW) sources. Multi-object near-infrared spectrographs on the world's largest telescopes (i.e., MOSFIRE, FLAMINGOS-2, MOIRCS, EMIR, KMOS, etc.) allow for the study of a much greater number of galaxies, but are still subject to the limitations of the available atmospheric transmission windows. More importantly, multi-object spectroscopy generally requires target pre-selection based on broadband photometry, creating a bias against discovery of emission-line galaxies with the most extreme EWs.

An alternative method to spectroscopy for identifying large numbers of $z>0.7$ emission-line galaxies are wide-field narrowband searches targeting the transmission windows in our atmosphere, e.g., HiZELS (Geach et al. 2008; Sobral et al. 2009, 2012, 2013) and the NEWFIRM H $\alpha$ Survey 
(Ly et al. 2011). These surveys have the advantage of very high sensitivity to emission lines $\left(\sim 1 \times 10^{-17} \mathrm{erg} \mathrm{s}^{-1}\right)$, but suffer from an inability to map outside their narrow redshift ranges, making them unfeasible for BAO studies. In addition, there is the danger of significant contamination from emission lines at different redshifts, a well-known issue from high-redshift Ly $\alpha$ emission line searches (Martin et al. 2008; Henry et al. 2012). For instance, the typical $\mathrm{H} \alpha /[\mathrm{O} \mathrm{III}] \lambda 5007$ ratio decreases with redshift (Ly et al. 2007; van der Wel et al. 2011; Domínguez et al. 2013). A carefully constructed experiment, with multiple photometric bands along with additional narrow bands targeting another expected line at the same redshift, can increase the narrowband reliability (e.g., Lee et al. 2012; Sobral et al. 2012), although surveys dependent on continuum detections will still miss the lowest mass galaxies.

Of course the best way to avoid the limitations of the atmosphere is to observe in space. Two future space missions, ESA's Euclid (Laureijs et al. 2012, 2011) and NASA's WFIRST (Green et al. 2012; Dressler et al. 2012), both utilize large-area, near-infrared grism surveys to investigate BAO (Glazebrook et al. 2005), as well as galaxy evolution and star formation history. In addition to identifying the redshift of galaxies, near-infrared spectroscopy also allows access to the wealth of stellar evolution diagnostic features available in the optical: $\mathrm{H} \alpha$, one of the most reliable indicators of star formation rate, the Balmer decrement, to determine extinction, as well as multiple metallicity indicators. These emission-line surveys will cover thousands of square degrees of continuous redshift space, unbiased by the underlying continuum luminosity of the galaxy. However, these will not be the first near-infrared grism observations done from space, as the Hubble Space Telescope (HST) has had two instruments with near-infrared grisms.

The Near IR Camera and Multi-Object Spectrometer (NICMOS; Thompson \& Schneider 1998) G141 grism pureparallels program (McCarthy et al. 1999; Yan et al. 1999; Shim et al. 2009) surveyed $\sim 170 \mathrm{arcmin}^{2}$, identifying 113 emitters and measuring the $\mathrm{H} \alpha$ luminosity function from $0.7<z<1.9$. The NICMOS data had a tiny $\left(<1 \operatorname{arcmin}^{2}\right)$ field of view, low spectral resolution $(R \sim 100)$, and relatively poor detector sensitivity. The Wide Field Camera 3 (WFC3; MacKenty et al. 2010) instrument is an improvement in all three areas, providing a 20-fold survey efficiency gain for the currently ongoing WFC3 Infrared Spectroscopic Parallels program (WISP; Atek et al. 2010, 2011). While much smaller in area, the WISP survey is much deeper than the future space near-infrared grism surveys. There is currently no better laboratory for predicting what these future missions can expect.

In this paper we discuss a sample of the WISP emissionline objects identified from $0.85-1.65 \mu \mathrm{m}$. We discuss the survey, details of the emission-line extraction, completeness corrections, and estimates of redshift accuracy. We then present the $\mathrm{H} \alpha$ and [O III] line-emitter number counts, $\mathrm{H} \alpha /[\mathrm{O}$ III] ratios, and luminosity functions. Finally, we present our final summary and conclusions. We assume an $\Omega_{M}=0.3, \Omega_{\Lambda}=0.7$ universe with $H_{0}=70 \mathrm{~km} \mathrm{~s}^{-1} \mathrm{Mpc}^{-1}$. All magnitudes are in the $\mathrm{AB}$ system.

\section{WISP SURVEY}

The WISP survey (PI: Malkan; GO-11696, GO-12283, GO-12568, and GO-12902) consists of HST WFC3 (Kimble et al. 2008) grism observations in uncorrelated high-latitude fields obtained in parallel mode while prime observations are being obtained with the Cosmic Origins Spectrograph
Table 1

Summary of WISP Field Observations

\begin{tabular}{|c|c|c|c|c|c|c|}
\hline Field & $\begin{array}{c}\text { R.A. } \\
\text { (HMS) }\end{array}$ & $\begin{array}{c}\text { Decl. } \\
\text { (DMS) }\end{array}$ & $\begin{array}{l}\text { G102 } \\
\text { (s) }\end{array}$ & $\begin{array}{c}\text { F110W } \\
(\mathrm{s})\end{array}$ & $\begin{array}{c}\text { G141 } \\
\text { (s) }\end{array}$ & $\begin{array}{c}\text { F160W } \\
(\mathrm{s})\end{array}$ \\
\hline Par17 & $02: 13: 38.11$ & $+12: 54: 59.3$ & 3409 & 534 & 3409 & $559^{a}$ \\
\hline Par55 & $12: 20: 54.68$ & $-02: 04: 46.0$ & 6415 & 909 & 2809 & $484^{\mathrm{a}}$ \\
\hline Par62 & $13: 01: 16.20$ & $-00: 00: 20.2$ & 4712 & 734 & 2006 & 396 \\
\hline Par64 & $14: 37: 29.04$ & $-01: 49: 49.5$ & 5918 & 1112 & 2306 & 456 \\
\hline Par68 & $23: 33: 33.04$ & $+39: 21: 20.5$ & 7721 & 1215 & 3009 & 534 \\
\hline Par69 & $15: 24: 07.75$ & $+09: 54: 53.9$ & 5721 & 1087 & 2309 & 431 \\
\hline Par73 & $14: 05: 12.86$ & $+46: 59: 19.9$ & 6118 & 1034 & 2509 & 456 \\
\hline Par74 & 09:10:48.14 & $+10: 17: 20.3$ & 5918 & 1065 & 2306 & 431 \\
\hline Par76 & $13: 27: 22.17$ & $+44: 30: 39.3$ & 5515 & 887 & 2006 & 406 \\
\hline Par78 & $23: 2834.06$ & $+05: 10: 28.3$ & 5318 & 887 & 2106 & 406 \\
\hline Par79 & 01:10:08.96 & $-02: 25: 16.2$ & 7521 & 1187 & 2809 & 534 \\
\hline Par81 & 01:10:09.12 & $-02: 22: 17.1$ & 7521 & 1187 & 2809 & 534 \\
\hline Par87 & $09: 46: 46.39$ & $+47: 14: 58.2$ & 4915 & 912 & 1906 & 406 \\
\hline Par94 & $22: 05: 26.66$ & $-00: 17: 48.5$ & 9024 & 1624 & 3309 & 534 \\
\hline Par96 & 02:09:24.40 & $-04: 43: 41.6$ & 28081 & 4295 & 11430 & 1765 \\
\hline Par97 & 01:10:06.30 & $-02: 23: 44.7$ & 5515 & 859 & 2109 & 406 \\
\hline Par114 & $10: 40: 58.09$ & $+06: 07: 31.0$ & 7221 & 1137 & 2909 & 456 \\
\hline Par115 & 11:18:55.08 & $+02: 17: 09.6$ & 5215 & 912 & 2106 & 381 \\
\hline Par120 & $13: 56: 51.50$ & $+17: 02: 33.9$ & 4512 & 837 & 1806 & 381 \\
\hline Par124 & $18: 32: 28.28$ & $+53: 44: 50.9$ & 4618 & 759 & 1906 & 406 \\
\hline Par129 & 11:02:18.72 & $+20: 52: 07.8$ & 4712 & 762 & 2206 & 456 \\
\hline Par131 & $10: 48: 22.94$ & $+13: 03: 50.5$ & 13039 & 2171 & 5215 & 884 \\
\hline Par132 & $11: 26: 19.80$ & $-01: 43: 22.1$ & 4315 & 634 & 1806 & 356 \\
\hline Par135 & $11: 22: 24.01$ & $+57: 50: 58.9$ & 4712 & 862 & 1906 & 406 \\
\hline Par136 & $12: 26: 28.84$ & $+05: 23: 02.9$ & 18857 & 3036 & 7318 & 1137 \\
\hline Par143 & $14: 02: 22.01$ & $+09: 45: 51.7$ & 10133 & 1568 & 4012 & 759 \\
\hline Par146 & $02: 12: 27.60$ & $-07: 32: 20.7$ & 4212 & 887 & 1706 & 381 \\
\hline Par147 & 23:58:19.51 & $-10: 15: 04.6$ & 5418 & 962 & 2106 & 406 \\
\hline Par167 & $01: 41: 24.18$ & $+13: 37: 34.1$ & 4315 & 659 & 1806 & 356 \\
\hline
\end{tabular}

Note. ${ }^{\text {a }}$ Used F140W as the direct image for identifying counterparts in the G141 grism.

(Osterman et al. 2011) or the Space Telescope Imager and Spectrograph (Woodgate et al. 1998). The parallel data include both grism spectroscopy and associated near-IR and optical imaging. Depending on the length of the parallel opportunity, the WISP survey either acquires spectroscopy with just the G141 (1.2-1.7 $\mu \mathrm{m}, R \sim 130)$ grism or, in the case of longer opportunities, a combination of G141 and the G102 $(0.8-1.2 \mu \mathrm{m}$, $R \sim 210$ ) grism with roughly $2-3$ times more integration time spent on the higher resolution G102 grism. At a plate scale of 0.13 pixel $^{-1}$, the total field of view for each observation is $123^{\prime \prime} \times 136^{\prime \prime}$. We note that one can only achieve the full grism wavelength resolution for compact sources, as any spatial extension of the object will also broaden the features within the spectrum. See Atek et al. (2010) for further discussion of the program.

For this paper we present data from 29 separate fields where we have both G102 and G141 grism spectroscopy, covering a total of $135 \operatorname{arcmin}^{2}\left(0.037 \mathrm{deg}^{2}\right)$ over 159 orbits. These fields, along with their integration times, are presented in Table 1. While other large G141-only grism surveys have been approved by HST (e.g., 3D-HST and AGHAST; Brammer et al. 2012; Weiner 2012), no other program comes close to surveying the area that WISP does with such an extended spectral coverage (0.85-1.65 $\mu \mathrm{m})$.

The inclusion of the G102 grism doubles the wavelength and redshift range surveyed. More importantly, it provides both 
critical checks of the assumed redshift and multiple line ratios that cannot be obtained using G141 alone. [O III] and $\mathrm{H} \alpha$ only fall together on the G141 grism over a very narrow redshift range $(z=1.3-1.55)$; otherwise, in the vast majority of cases G141only observations only discover single emission lines. [O III] lines of comparable or greater strength than $\mathrm{H} \alpha$ are common (Hu et al. 2009; Atek et al. 2010; Domínguez et al. 2013), making a catastrophic misidentification likely. With both G102 and G141, WISP is sensitive to $\mathrm{H} \alpha$, [O III], $\mathrm{H} \beta$, and [O II] over a wide range of redshifts, and for $z=1.3-1.55$, we are sensitive to all those lines simultaneously.

\section{EMISSION-LINE EXTRACTION}

All the data were processed with the WFC3 pipeline CALWF3 (version 2.1) to correct for bias, dark, flat-field, and gain variations. Then, the slitless extraction package aXe 2.0 (Kümmel et al. 2009) is used for the spectral extraction. A complete description of the data reduction steps is presented in Atek et al. (2010).

To locate and identify all the emission lines within our spectra, we applied an automatic line-finding algorithm. We first fit a three-segment cubic spline to the continuum of our onedimensional aXe-extracted spectra, using outlier rejection to avoid fitting any emission lines. We then subtract the estimated continuum from the spectrum and divide the continuumsubtracted spectrum by the aXe estimate of the flux uncertainties for each pixel. This produces a $\mathrm{S} / \mathrm{N}$ ratio $(\mathrm{S} / \mathrm{N})$ spectrum. We look for groupings of three or more contiguous pixels with an excess above the continuum with $\mathrm{S} / \mathrm{N}$ in each pixel greater than $\sqrt{3}$. For an unresolved object, the emission lines can be as narrow as two pixels, and so we adopt a more stringent two-pixel criterion of two contiguous pixels with an $\mathrm{S} / \mathrm{N}$ of greater than $\sqrt{5}$ in each. To validate this method of finding candidate emission lines, we compared several samples of emission lines found automatically to those found by an intensive, spectra-by-spectra, visual inspection and found that the automated method did not miss any significant fraction of lines $(<5 \%)$.

The parallel nature of the WISP observations presents several challenges to emission-line extraction. Foremost is the lack of dithering, which greatly complicates the mitigation of cosmic rays, hot or warm pixels, and other artifacts. As a practical matter, this means that the automated line identification process has a very high contamination rate from false and/or spurious sources.

To address the false detections, we require the visual inspection of every candidate emission line by two team members, done independently and without consultation. After this initial inspection, we compare the emission-line lists from the two reviewers and send each reviewer back a list of discrepancies, allowing them to make a second, more careful examination of any emission lines for which there is a disagreement. This exercise provides a good final agreement for most emission lines, but we find that for roughly $5 \%$ of the emission lines, consensus cannot be reached. These uncertain lines are removed from the final sample, but are accounted for in our completeness corrections (see Section 3.1).

The visual inspection consists of examining both the extracted one-dimensional and the original two-dimensional spectra, as well as the continuum fits and measurements of $\mathrm{S} / \mathrm{N}$. There are only four conditions for which we exclude a candidate line:
1. If the line is clearly an artifact, either a cosmic ray, a zeroorder image from a nearby bright source, a diffraction spike, a ghost, or some other artifact clearly not associated with the spectrum in question.

2. If the continuum fit under the location of the emission line is highly inaccurate, artificially increasing the significance of the emission line. Many of the spectra contain breaks, both real and caused by nearby contamination from overlapping sources, which often lead to continuum errors of this sort.

3. If the local noise variations are larger than the flux uncertainties used by the automated line identification software, measuring lines at a higher significance than local noise conditions actually warranted. While the WFC3 detector and grism are fairly uniform and well behaved, we find variations across the detector and locations where what appear to be non-Poissonian sources of noise exist. In these cases the automated recovery pipeline was likely to find every noise peak and call it a source.

4. If the contamination from nearby overlapping spectra is so great that we cannot determine what source is producing the emission line. However, in most cases even severe overlapping source contamination can be untangled. We use multiple lines spread across the G102 and G141 grisms (only the real source produces the correct wavelength ratios in those cases), small pixel offsets from source center, and discrepancies between the size of the object and the spatial extent of the emission line as evidence for assigning the confused emission line to its proper source. Only if the source remains ambiguous after all analysis is it excluded from the final sample.

Single emission lines are assumed to be $\mathrm{H} \alpha$, except in the few rare circumstances where the single line is clearly resolved into the $[\mathrm{O}$ III $] \lambda \lambda 5007+4959$ doublet. Our simulations indicate that this is a good assumption and does not result in more than $6 \%$ of our $\mathrm{H} \alpha$ lines being actually misidentified [O III] (see Section 3.3 below). However, this assumption does have a large effect on the recovery of high-redshift $(z>1.5)$ [O III] lines, requiring some additional completeness corrections for those emission lines (see Section 3.1.1).

In total, we extracted 1960 emission lines from the 29 fields down to a typical flux limit of $(3-5) \times 10^{-17} \mathrm{erg} \mathrm{s}^{-1} \mathrm{~cm}^{-2}$. For an emission-line galaxy to be included in our final analysis, we also require an observed EW greater than $40 \AA$ (log $\mathrm{EW}=1.6$; see Section 3.1 below) and an $\mathrm{S} / \mathrm{N}$ greater than 5 for at least one of the detected emission lines, creating a final sample size of 1048 galaxies. Of these, 467 have multiple detected emission lines, including weaker [O II], $\mathrm{H} \beta$, and [S II]. For our final sample of emission-line galaxies, we detect $\mathrm{H} \alpha$ in 996 galaxies and [O III] in 280 galaxies. The overlap where we found both $\mathrm{H} \alpha$ and [O III] in the same galaxy is 226 .

\subsection{Completeness Corrections}

Virtually all surveys suffer incompleteness from objects lost as the strength of their signals approaches the level of the noise. A slitless grism survey, like WISP, also suffers significant incompleteness due to confusion from nearby bright sources. In addition to those two fundamental sources of incompleteness, emission lines can also be lost as part of the extraction methodology. These sorts of failures include objects missed by the automatic line-finding routine, objects removed due to redshift confusion, and objects incorrectly rejected by visual inspection. In order to derive final completeness 

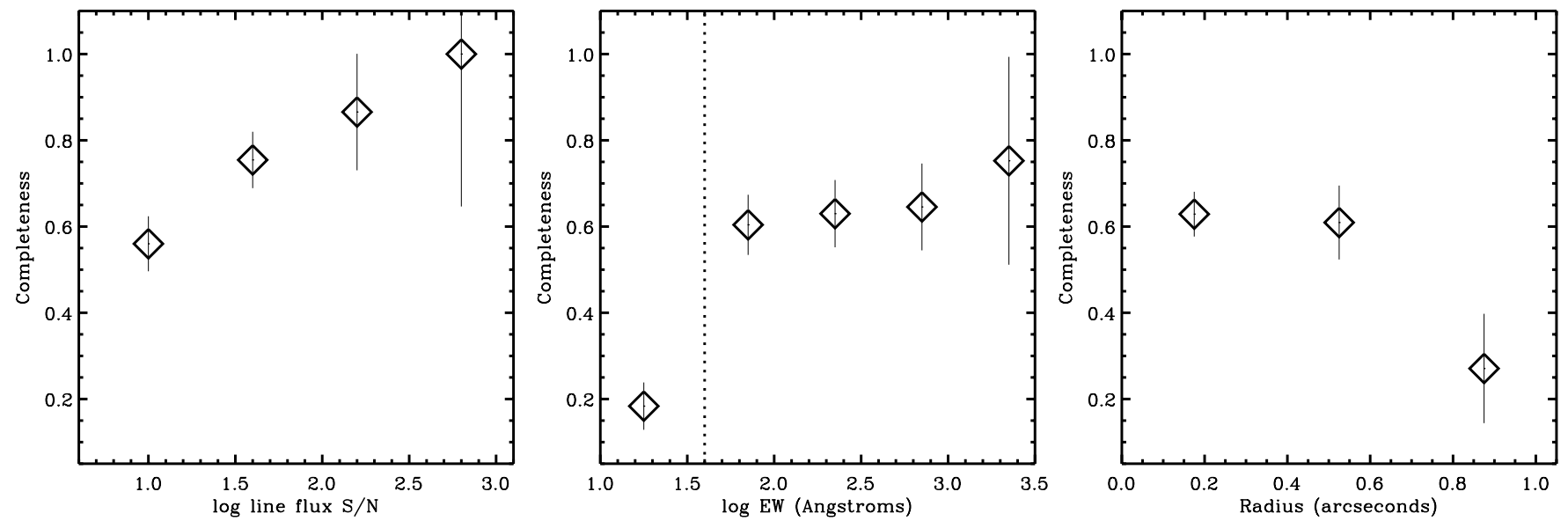

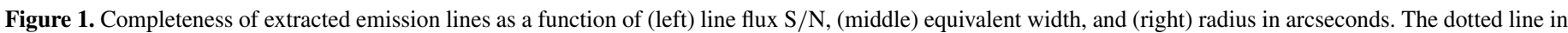
the middle equivalent width plot represents the chosen equivalent width cut.

corrections to account for all the forms of incompleteness that affect the grism data, we must simulate the entire line extraction process from beginning to end.

We start with the generation of a set of emission-line galaxy models, covering the full range of redshifts $(z=0.3-2)$, brightnesses (19-26 F110W mag), spatial extent $\left(0.2^{\prime \prime}-1^{\prime \prime}\right.$ radius), and EWs (20-2000 $\AA$ ) found in our WISP data set of emissionline galaxies. We have used two different spectral templates extracted from the Kinney-Calzetti Atlas in the STSDAS/ SYNPHOT library. The spectra are from real starburst galaxies observed over the whole UV and optical range up to $1 \mu \mathrm{m}$. We have chosen two spectra with relatively flat continuum in $f_{v}$ and different line ratios of $\mathrm{H} \alpha /[\mathrm{O}$ III $] \sim 4.3$ and 0.75 . The sizes of the galaxies were then simulated using random values of the minor and major axis defined as the FWHM of the light profile in the SExtractor catalog. Throughout this paper any reference to galaxy size or galaxy radius always refers to the size of the direct (F110W) image and not to the spatial extent of the emission lines seen in the grism spectra. We place each model galaxy randomly into one of our actual pairs of WISP G102 and G141 grism images using the aXeSIM software (Kuemmel et al. 2007), before extracting it using the same methodology and pipeline as used for extracting all the real emission-line galaxies. This means that first we identify the lines using the automated software and then two separate team members visually examine each spectrum to ensure that it contains a real emission line. During the visual inspection we mix 15 model galaxies along with another 15 randomly chosen galaxies, such that the reviewer could not assume that each spectrum must contain a visible emission line. Overall we insert 923 model galaxies (74 fields each with typically 10-15 model galaxies) and, including the random galaxies, extract twice that number.

We find that the primary determinants of completeness for the emission-line galaxy sample are the observed EW and the line flux of the emission line. In practice, the latter translates to the $\mathrm{S} / \mathrm{N}$ of the emission-line flux measurement, as the background noise varied from field to field by a factor of four. We also find that completeness had some dependency on the radius (here defined as the major and minor axis determined by SExtractor averaged in quadrature) of the source galaxy, but that this is of secondary importance due to the relatively small number of large $(>0.75)$ radius galaxies in the observed sample, approximately $0.5 \%$ (see Section 4.2 for further discussion of the size of emission-line galaxies).
In Figure 1, we plot completeness as a function of these three parameters (line flux $\mathrm{S} / \mathrm{N}, \mathrm{EW}$, and size), where each bin is a combination of recovery rates for the model galaxies. We weight all the model galaxy recovery rates for each single parameter by the frequency in which galaxies with the other two parameters appear in our full sample of actual emissionline galaxies. We acknowledge that these observed frequencies of the different galaxy parameters are not the absolute true frequencies. However, the implied differential numbers of "missing" emission-line galaxies due to incompleteness are not large enough to make a significant impact on the final derived incompletenesses, so we weight by the observed parameter frequencies in the interest of simplicity. For instance, if the full sample has twice as many small EW objects as large EW objects, model galaxies with small EWs will be given twice the weight when deriving completeness for $\mathrm{S} / \mathrm{N}$ or size.

One immediate result from this simulation is that the recovery rate drops rapidly from $60 \%$ to $<20 \%$ as the observed EW $(\AA)$ of the emission line becomes less than $40 \AA(\log \mathrm{EW}=1.6$; see Figure 1). In the rest frame of our $z \sim 1$ galaxies, this EW limit corresponds to $20 \AA$, roughly two-thirds that of the average $\mathrm{H} \alpha$ EW for spiral galaxies in the Local Volume (Lee et al. 2007) and approximately equivalent to the average $\mathrm{H} \alpha \mathrm{EW}$ for the most massive $z=0.8-1.5$ galaxies $\left(11<\log M_{\odot}<11.5\right)$ identified by 3DHST (Fumagalli et al. 2012). In the interest of not introducing extremely large $(>5)$ and uncertain completeness corrections to the number counts, we apply a cut of EW > $40 \AA$ to all of our analysis. After combining this EW cut with our requirement that all detected emission lines have an $\mathrm{S} / \mathrm{N}$ $>5$, the total number of model galaxies is 553. Of those model galaxies, we recovered 380 , yielding an approximate recovery rate of $69 \%$. We note that for this combination of EW and $\mathrm{S} / \mathrm{N}$, there are effectively no galaxies fainter than $25 \mathrm{mag}$ (as measured at $\mathrm{F} 110 \mathrm{~W}$ band, although the simulated galaxies are flat in $F_{v}$ ) in the final sample we use for our analysis.

In Figure 2, we present emission-line EW as a function of $\mathrm{S} / \mathrm{N}$ for both observed and model emission-line galaxies. One can see that the model galaxies span the same parameter range as the real galaxies, extending farther to brighter lines with large EWs and fainter lines with smaller EWs in order to test how our sample completeness acts at these extremes. One complication is that over a significant portion of the redshift range $(z=0.7-1.5)$, there are actually two strong emission lines, [O III] and $\mathrm{H} \alpha$. In some cases [O III] is the more powerful line, particularly as we 


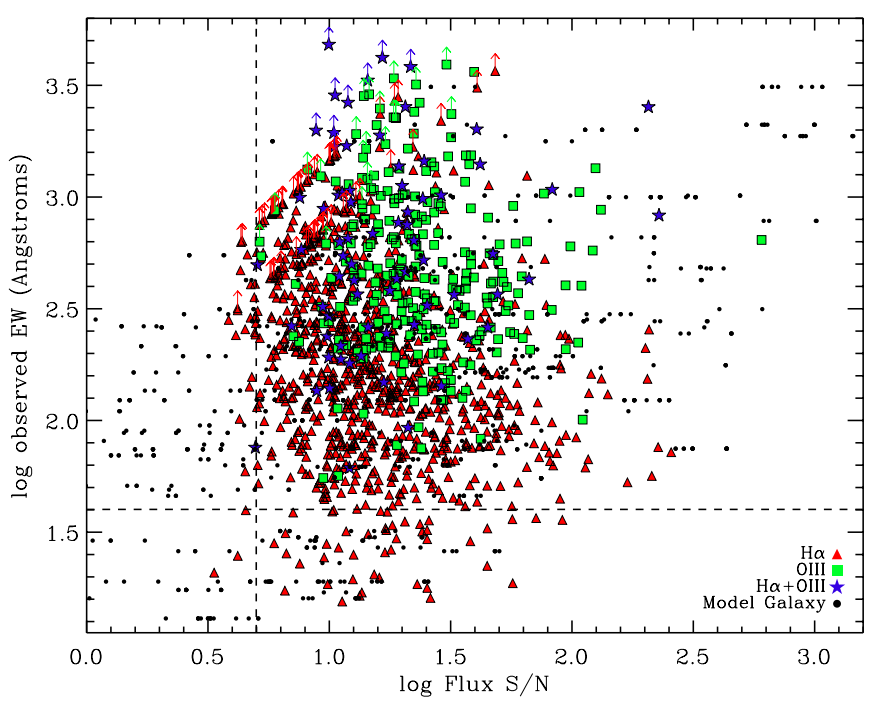

Figure 2. Plot of emission-line flux $\mathrm{S} / \mathrm{N}$ vs. equivalent width for all the identified WISP emission lines. Red triangles are objects with $\mathrm{H} \alpha$ emission lines, green squares are objects with [O III] emission, and blue stars are objects where both lines are detected. Where both $\mathrm{H} \alpha$ and $[\mathrm{O}$ III] are detected, the parameters plotted are sums $(\mathrm{EW})$ or quadratic sums $(\mathrm{S} / \mathrm{N})$ of the two lines together. Overplotted as solid black circles are the model emission-line galaxies used for our completeness simulations. The dashed lines are the limits in flux and EW applied to our sample. Emission lines that lie beyond those limits have been excluded from the final analysis.

(A color version of this figure is available in the online journal.)

go to fainter line fluxes (see Section 4.2 below). We therefore used both model templates where $\mathrm{H} \alpha$ is more powerful and ones where $[\mathrm{O} \mathrm{III}]$ is the stronger emission line $(\mathrm{H} \alpha /[\mathrm{O} \mathrm{III}]=$ 0.75-4.3). We plot the redshift distributions for the detected $\mathrm{H} \alpha$ and [O II] emitters in Figure 3. The gap in the $\mathrm{H} \alpha$ distribution around $z=0.8$ is a result of the low $\mathrm{S} / \mathrm{N}$ wavelength range edges of the G102 and G141 grisms where they overlap at 1.1-1.2 $\mu \mathrm{m}$.

One would expect a source with multiple lines to have a higher completeness than a single line source with the same line flux and EW. Therefore, to determine the likelihood of identification, we measure the combined $\mathrm{S} / \mathrm{N}$ of both lines, add them quadratically, and use that total $\mathrm{S} / \mathrm{N}$ for calculating our completeness. For the combined EW we use a simple sum of the two emission lines. We plot these combined values of $\mathrm{S} / \mathrm{N}$ and EW as blue stars in Figure 2, along with the single-line cases of $\mathrm{H} \alpha$ (red triangles) and [O III] (green squares). In most cases one line is clearly stronger, so the final $\mathrm{S} / \mathrm{N}$ used for the completeness correction can usually be approximated as the $\mathrm{S} / \mathrm{N}$ of just the brightest emission line. We do not use the other, weaker emission lines ([O II], [S II], $\mathrm{H} \beta$, etc.) for determining completeness, as these lines are never alone and their relative fluxes are almost always small compared to $\mathrm{H} \alpha$ and [O III].

We find that for even the highest $\mathrm{S} / \mathrm{N}$ lines $(\mathrm{S} / \mathrm{N}>100)$, we reach only $90 \%-95 \%$ completeness. The main reason for this is contamination, from either overlapping spectra or large, bright objects in the direct images used to identify the galaxy counterparts. Either the line is lost under an extremely bright spectrum (most fields have three to five moderately bright $H<$ 19 objects), the object lies in a complicated region where we are unable to determine which of the nearby, overlapping objects to associate with the emission line, or the contamination from overlapping sources has caused the automatic line finder to fail, i.e., SExtractor fails to find the source or the continuum fit fails badly. Altogether contamination from nearby sources explains about $75 \%$ of line extraction failures. Most nearby source

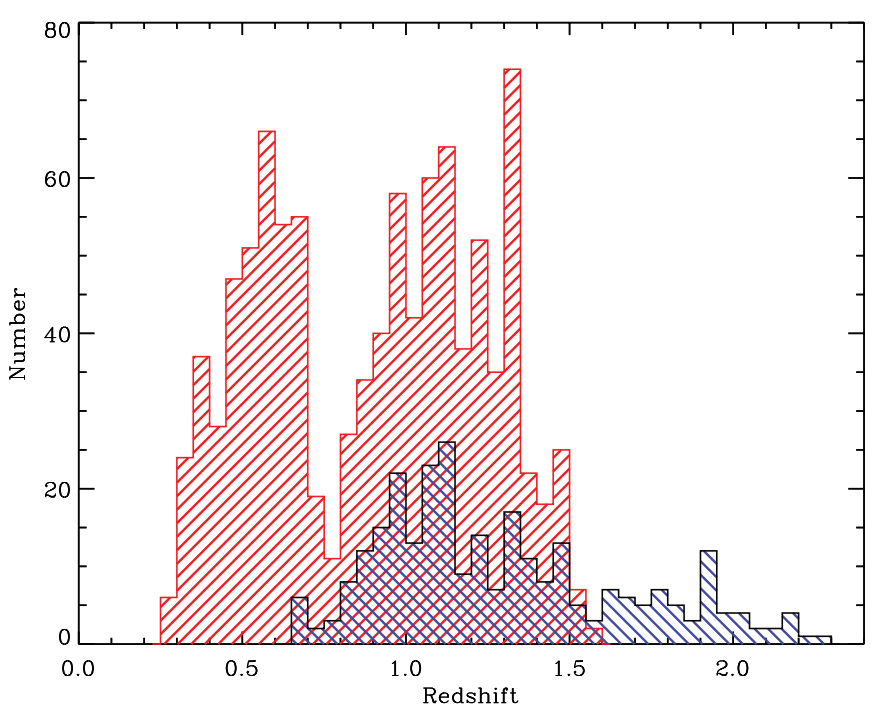

Figure 3. Histogram of redshifts for all emission-line objects. The larger distribution (red) is objects with $\mathrm{H} \alpha$ emission, while the smaller distribution (blue) is objects with [O III] emission. Objects with both $\mathrm{H} \alpha$ and [O III] emission are included within both distributions and can be approximated as the region where the two distributions overlap (there are only two $z<1.5$ [O III] emitters without an $\mathrm{H} \alpha$ detection).

(A color version of this figure is available in the online journal.)

contamination issues would be resolved if data could be taken at two or more roll angles, such that the spectra do not overlap, but that is not an option for parallel programs. Another reason a strong line will fall out of our sample is if the two emissionline reviewers cannot come to consensus on the redshift of the object ( $10 \%$ of the bright extraction failures), which most commonly occurs where it is unclear whether the line is $\mathrm{H} \alpha$ or [O III]. For instance, when a source is spatially extended, the grism wavelength resolution is effectively lowered, making it difficult to distinguish between the [O III] doublet and a single emission line. This sort of confusion almost never occurs in G102 $(R \sim 210)$, suggesting that data taken with a higher spectral resolution would largely solve this problem as well. The remaining $\sim 15 \%$ of bright emission line failures are mostly miscellaneous extraction errors by the visual line inspectors.

In Figure 4, we plot the final completeness corrections used for all of our analysis, derived as a function of both the EW and the $\mathrm{S} / \mathrm{N}$ of the line flux. While the object size also has an effect on whether an emission-line galaxy will be recovered, we found that accounting for galaxy size has little effect on the overall completeness corrections, as the largest galaxies make up only a tiny percentage of the sample, $<2 \%$, even after accounting for their higher incompleteness. Or to put it another way, galaxy size is not very important to the overall completeness correction, because size completeness corrections grow larger as the actual number of galaxies is decreasing, unlike EW or line flux, where the number of objects tends to grow just as their completeness corrections are significantly increasing. A three-parameter completeness correction is also not practical for the required double visual inspection of each suspected emission line. Each added parameter increases the human workload geometrically. Instead, for each bin of EW and line flux $\mathrm{S} / \mathrm{N}$, we weight the input model galaxies by the frequency that each galaxy size appears in our observed sample list of emission-line galaxies. 


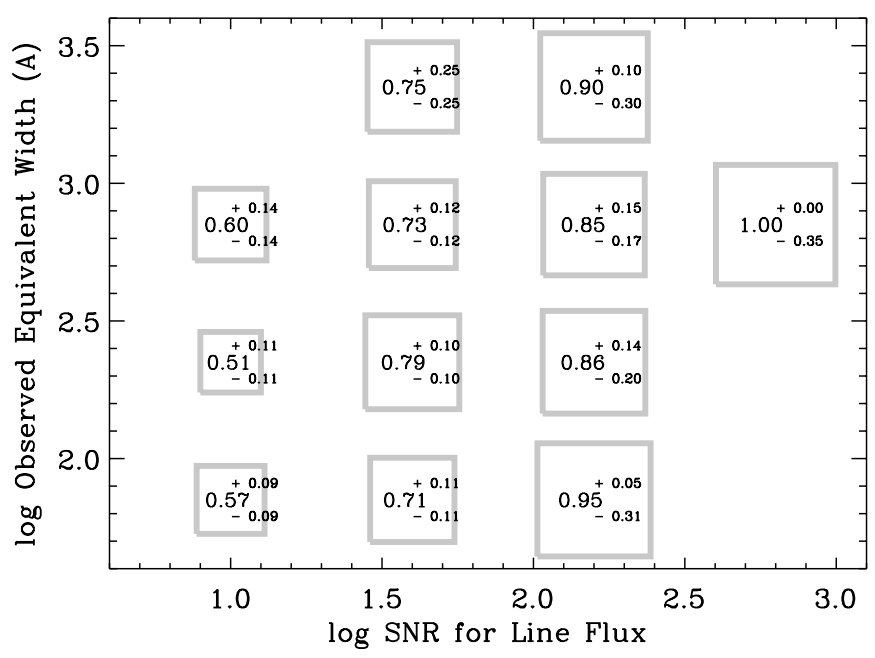

Figure 4. Diagram of completeness as a function of both line flux $S / N$ and equivalent width. Each box represents one of the two parameter bins used, with the measured completeness ratio number inside it, including its error. The larger the square box, the smaller the completeness corrections that were applied for those emission-line galaxies.

\subsection{1. [O III] Completeness}

Generally we found that the completeness corrections we derived for $\mathrm{H} \alpha$ can also be applied to [O III] emission lines. While [O III] is a doublet with a distinctive line profile that one might expect could be more easily distinguished from artifacts and noise, for low $\mathrm{S} / \mathrm{N}$ and small EWs where the incompleteness becomes increasingly important, [O III] becomes indistinguishable from a single emission line.

This assumption that $\mathrm{H} \alpha$ and [O III] completeness can be treated the same breaks down for [O III] at redshifts of $z>1.5$. This is a result of the attribution of all single-line emitters to $\mathrm{H} \alpha$. Below $z=1.5, \mathrm{H} \alpha$ remains in the wavelength range that is detectable by the WFC3 grisms, and in the vast majority of cases if we detect an [O III] emission line, the $\mathrm{H} \alpha$ emission line will be detectable as well. This is because even down to our faintest emission line detections, the $\mathrm{H} \alpha /[\mathrm{O}$ III $]$ ratio is usually greater than 1 and rarely less than 0.4 (see Section 4.2). Above $z=1.5$, the $\mathrm{H} \alpha$ emission line is redshifted out of our detectable wavelength range, so we lose the ability to confirm [O III] with the usually brighter $\mathrm{H} \alpha$ line. If the [O III] line is bright enough, we can still identify it from its doublet line profile, but the faint [O III] lines will all be identified as lower redshift $\mathrm{H} \alpha$ lines by default.

While this misidentification of high- $z$ [O III $]$ produces only a small contamination of $\mathrm{H} \alpha$ (see Section 3.3), our simulations show we lose nearly $50 \%$ of our high- $z$ [O III] emission lines because of our inability to resolve the doublet. More specifically, the fainter the emission lines, the larger the incompleteness. We have therefore added an additional completeness correction to our $z>1.5$ [O III] emission lines of 2.0 for $0.6<\log \mathrm{S} / \mathrm{N}<1.2$ and 1.6 for $1.2<\log \mathrm{S} / \mathrm{N}<1.8$. Higher $\mathrm{S} / \mathrm{N}[\mathrm{O}$ III] emission lines require no additional correction.

\subsection{Redshift Accuracy}

Using the simulations, we can also get an estimate of the redshift accuracy that is possible with grism spectroscopy of this wavelength resolution. Each emission-line source is placed at a random position in the field, which introduces the same uncertainties on the wavelength solution as seen in real data, including unknown absolute positions and inexact object sizes.

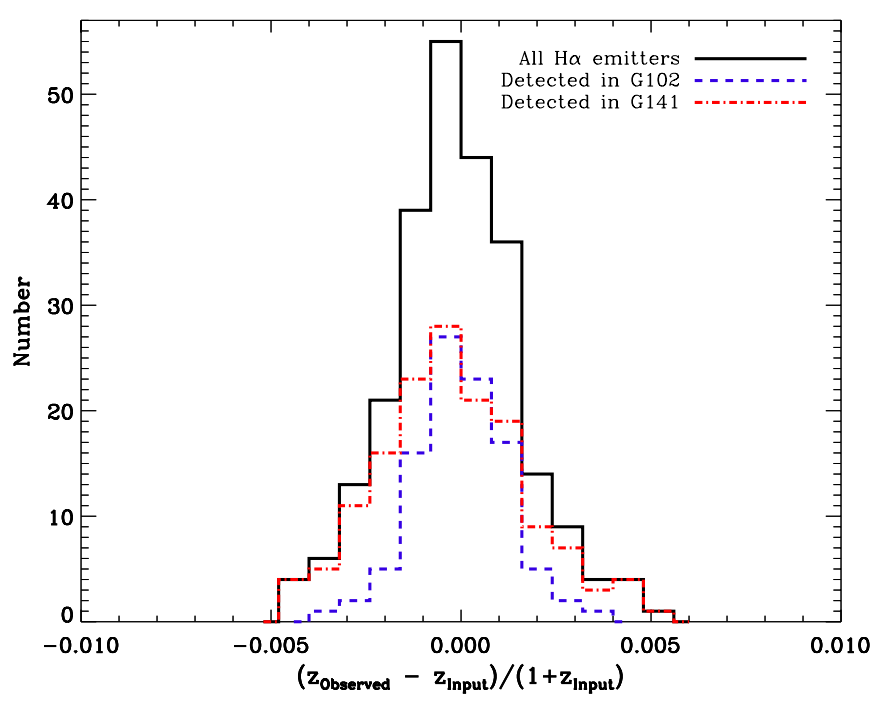

Figure 5. Histogram of redshift accuracy for $\mathrm{H} \alpha$ emitters taken from the simulations, where the accuracy is defined as $\left(z_{\text {observed }}-z_{\text {input }}\right) /\left(1+z_{\text {input }}\right)$. The solid black histogram is all $\mathrm{H} \alpha$ emitters, the dashed red histogram is those emitters found only in the G102 grism, and the dot-dashed blue histogram is the emitters found only in the G141 grism.

(A color version of this figure is available in the online journal.)

While this is not a measurement of the absolute wavelength accuracy-the model spectra are put in and taken out with the same software (aXe) and the same assumed wavelength solution-it is a reasonable test of the accuracy one can expect with this pixel sampling, extraction method, and $\mathrm{S} / \mathrm{N}$.

We removed the emission lines flagged because the reviewers could not agree on a redshift and then applied our $\mathrm{S} / \mathrm{N}$ cut of 5 and EW cut of $40 \AA$. We found that going to even higher $\mathrm{S} / \mathrm{N}$ and larger EW made almost no difference in the redshift accuracies we derived. For this analysis we are not interested in the catastrophic errors, which represent contamination of false lines in our samples rather than a real reflection of redshift accuracy, so we removed any objects with errors in $1+z>0.5 \%$. We discuss issues of contamination of false sources in Section 3.3. We further note that there is an error in the aXe process of inserting and removing user-generated spectra that incorrectly shifts the output wavelength calibration by a single pixel, corresponding to $24 \AA$ in G102 and $46 \AA$ in G141, which we have removed from all further analysis.

In Figure 5 , the histogram of $\left(z_{\text {observed }}-z_{\text {input }}\right) /\left(1+z_{\text {input }}\right)$ shows that the results are strongly peaked around zero. The median offset is -0.0002 with a standard deviation of 0.0017 , or roughly $0.2 \%$ accuracy in redshift. Modeling of BAOs suggests that the redshift accuracies required for such an experiment are more like $0.1 \%$ (Wang et al. 2010), which is also the requirement for both the Euclid and WFIRST missions. However, it is important to recall that the WISP redshifts are a combination of two different near-infrared grisms (G102 and G141) with two different resolutions $(R \sim 210$ and $R \sim 130)$. If we split the redshift accuracy histograms by the grism from which it came, we find that the higher resolution grism reaches accuracies of $0.13 \%$ (as opposed to $0.19 \%$ in G141), demonstrating that $0.1 \%$ redshift accuracy is possible if the wavelength resolution $R>200$.

It is important to note that the lack of sub-pixel dithering of the parallel observations prevents any meaningful resampling of pixels along the direction of the wavelength dispersion. Such resampling would provide a small improvement in the overall 
wavelength resolution and would therefore also potentially increase the redshift accuracies that are possible.

\subsection{False Emission Line Contamination}

Identifying emission lines in grism spectroscopy fields without dithering or field rotation is a challenging task. Even the well-behaved WFC3 near-infrared arrays still have cosmic rays, hot pixels, and other phenomena that can mimic real emission lines. Therefore, as we approach the detection threshold there is a real chance of contamination of our $\mathrm{H} \alpha$ line sample by false emission lines. To investigate this further, we look again to the simulation data. For the completeness calculation, we track whether an emission-line galaxy is detected and placed into our final emission-line list. There is no requirement that input and output redshifts must match. A mismatched redshift indicates contamination. While an emission line was found, it is clearly not identical to the emission line that was input. We label all extracted simulated spectra with a difference between input and output $(1+z)$ of more than $1 \%$ as contamination.

Of the $364^{12}$ simulated $\mathrm{H} \alpha$ emission lines that meet all extraction criteria $(\mathrm{S} / \mathrm{N}>5, \mathrm{EW}>40 \AA), 312$ are good emission lines found at the input redshift, a successful extraction rate of $85.7 \%$. An additional 21 emission lines are at the correct input redshift if we assign them to be [O III] rather than $\mathrm{H} \alpha$, for a contamination of $\mathrm{H} \alpha$ emission lines by [O III] of $5.8 \%$. For the remaining lines there are no alternative emission lines with which they could be associated, so they are contamination of non-emission lines to the sample. That gives us two contamination rates for our sample: $8.5 \%$ contamination by false emission lines and $14.3 \%$ total contamination rate of $\mathrm{H} \alpha$ emission lines by the combination of [O III] and false emission lines. These contamination rates are similar to the 15\%-30\% contamination rates found for narrowband $\mathrm{H} \alpha$ searches (Ly et al. 2011; Sobral et al. 2013), although we note that additional information (e.g., galaxy colors, photometric redshifts, and especially the use of a second narrowband filter) can reduce the narrowband contamination rate down to 5\% (Lee et al. 2012; Sobral et al. 2013).

This false emission line contamination rate decreases slightly as we increase the cut-offs of either $\mathrm{EW}$ or $\mathrm{S} / \mathrm{N}$ of the line fluxes, but contamination rate never improves to much less than $10 \%$. For instance, whether we raise the EW cut-off from $40 \AA$ to $200 \AA$ or raise the $\mathrm{S} / \mathrm{N}$ line flux cut-off from 5 to 20 , the false emission line contamination rate drops by only $3.5 \%$, to $10.8 \%$ total contamination.

We note that one other potential contaminant is [O II $] \lambda 3727$. [O II] emission is generally faint, with fluxes around $40 \%$ those of [O III $\lambda \lambda 5007+4959$. For redshifts where [O II] and [O III] both fall into the detectable near-infrared wavelength range $(1.3<z<2.3)$, if we detect [O II], we always detect [O III] as well. There are some rare cases where [O II] $\lambda 3727$ can be as much as $30 \%$ brighter than [O III], but that [O II]/[O III] ratio is not large enough to make any significant number of [O III] emitters undetectable. Therefore, we do not expect any contamination from [O II] $\lambda 3727$ below a redshift of $z<2.3$. However, the assumption that all single emission lines are $\mathrm{H} \alpha$ could potentially lead us to miss a population of very high redshift $(z>2.3)$ [O II] emitters. These high-redshift [O II] emitters would have to be very luminous, i.e., more than five

\footnotetext{
12 This number is slightly less than the 380 simulated emission lines referred to in Section 3.1, as it does not contain 16 weak $\mathrm{H} \alpha$ emitters that were found only because of their [O III] emission.
}

times the $L_{\star}$ found for O II at $z=1.47$ (Ly et al. 2007), and should therefore be quite rare. By $z=2$, the number density of [O II] emitters detectable by the WISP survey has dropped below $2 \times 10^{-4} \mathrm{Mpc}^{-3}$. If we allow for a simple luminosity evolution from $z=1.5-2.5$ that is consistent with the observed $z=2\left[\mathrm{O}\right.$ II] number density (a $0.2 \mathrm{mag}$ increase in $L^{*}$ ), then we would not expect more than a dozen $z>2.3$ [O II] emitters hidden in our $\mathrm{H} \alpha$ sample.

We do not make any separate corrections for these false emission line contamination rates in our analysis. The completeness corrections we apply are based on all the extracted emission lines from the simulations, including the contaminating lines. While the contaminating lines artificially inflate the number of model emission-line galaxies recovered, which reduces the derived completeness corrections, we expect the same contamination rates in the actual data. If we used completeness corrections that did not include contaminating lines, we would incorrectly produce final number counts that were too large. By leaving the contaminating lines in our completeness derivation, we produce completeness corrections that simultaneously account for the effects of both incompleteness and contaminating lines. To put it another way, the completeness corrections we use are $\sim 15 \%$ smaller than they would otherwise be because of the extra emission lines we added due to contamination.

\section{EMISSION-LINE NUMBER COUNTS}

The most numerous emission line seen by far is that of $\mathrm{H} \alpha$, which makes up two-thirds of all those observed. Of the $996 \mathrm{H} \alpha$ emission lines with EWs $>40 \AA$ and $\mathrm{S} / \mathrm{N}>5$, $577(58 \%)$ are identified from a single line. While identifying $\mathrm{H} \alpha$ emission from a single line introduces the possibility of a misidentification, our simulations (see Section 3.3) demonstrate that this affects no more than $6 \%$ of our emission-line galaxies. Since virtually all the misidentifications are single emission lines, this implies that roughly $10 \%$ of our single $\mathrm{H} \alpha$ emission lines are actually not $\mathrm{H} \alpha$ at all, but instead misidentified [O III]. While far from negligible, the level of [O III] emission line contamination down to the flux levels probed by this work $((3-5)$ $\times 10^{-17} \mathrm{erg} \mathrm{s}^{-1} \mathrm{~cm}^{-2}$ ) remains small enough (10\% of single emission lines, $6 \%$ of all lines) that it can be easily accounted for in the statistical analysis.

In Figures 6 and 7, we plot the sky density for $\mathrm{H} \alpha$ and [O III] versus flux, EW, and redshift in both the original raw counts and those corrected for completeness. Here we can see that there are significant completeness corrections applied across the entire range of fluxes.

\subsection{Emission-line Number Densities}

To derive the number density of the $\mathrm{H} \alpha$ emission lines in our survey, we employed the $V_{\text {MAX }}$ method (Felten 1977), in which we derive the maximum distance for which an emission line of that absolute luminosity could be detected, depending on the flux limits of each field, and then translate that to a volume, $V_{\mathrm{MAX}}$. This can be translated into a density:

$$
\delta_{\mathrm{gal}}=\frac{4 \pi}{\Omega} \sum C(\mathrm{~S} / \mathrm{N}, \mathrm{EW}) \frac{1}{V_{\mathrm{MAX}}},
$$

where $C(\mathrm{~S} / \mathrm{N}, \mathrm{EW})$ is the completeness correction for each detected emission-line galaxy, as a function of $\mathrm{S} / \mathrm{N}$ and equivalent width. We scale the volume of the entire sphere of the sky by the angular area observed, $4 \pi / \Omega$, to reach the volume density 

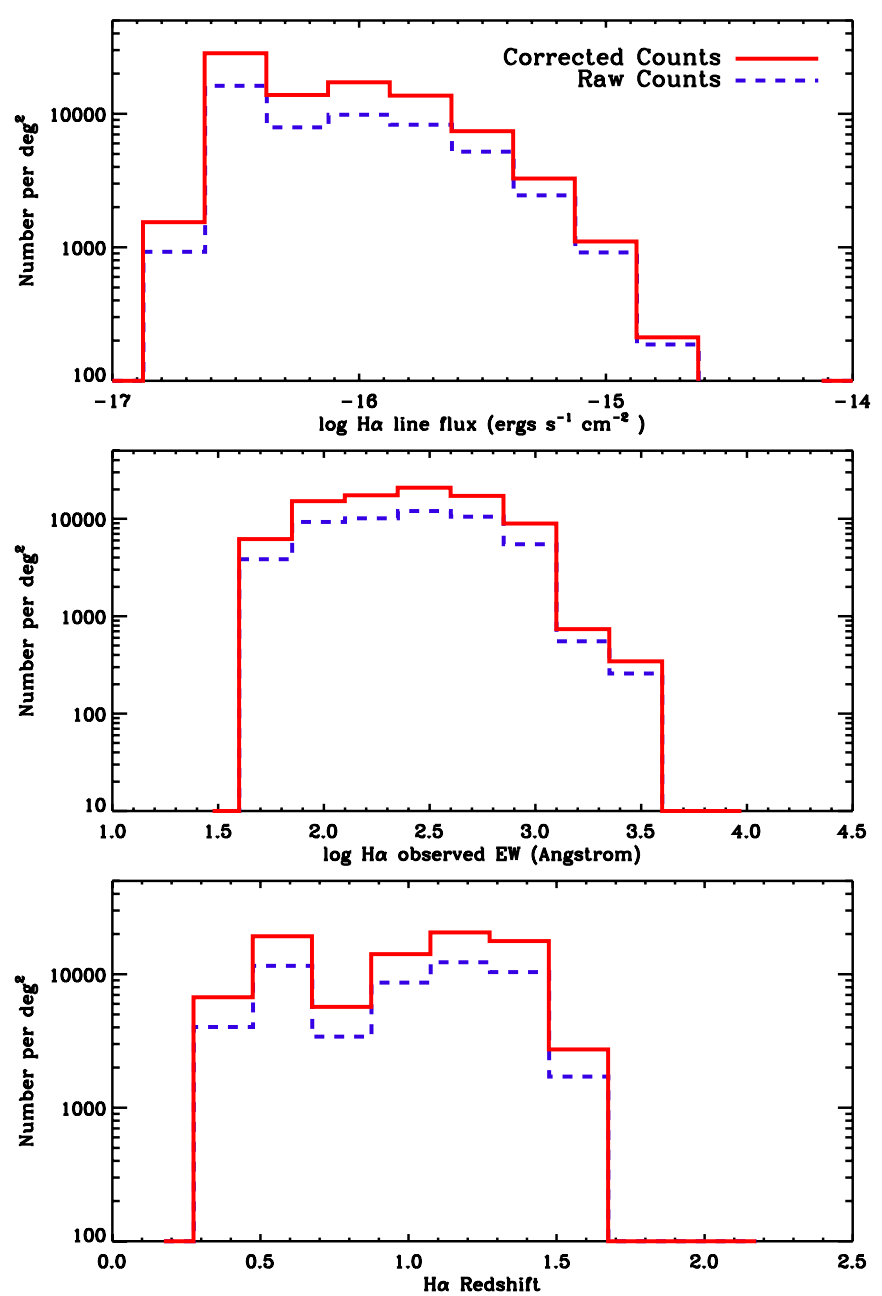

Figure 6. Histogram of all detected $\mathrm{H} \alpha$ line emitters as a function of (top) line flux, (middle) observed equivalent width, and (bottom) redshift. The dashed blue lines are the raw, uncorrected counts, while the solid red lines are the counts after the correction for completeness.

(A color version of this figure is available in the online journal.)

for each object, $\delta_{\text {gal }}$. Then we add up all the $\delta_{\text {gal }}$ to get the total volume densities, which we then use for derivation of the luminosity functions (see Section 4.3).

Similarly, we also calculate area number densities, deriving an $A_{\mathrm{MAX}}$ for each source. $A_{\mathrm{MAX}}$ is the total angular area from the survey for which the emission-line flux is greater than that of the $5 \sigma$ line limit of the field. For the brighter emission lines, $A_{\mathrm{MAX}}$ will simply equal the angular area of the entire survey (29 fields in this case). Similar to the $V_{\text {MAX method, once the }}$ $A_{\text {MAX }}$ values are determined, we add up all the $1 / A_{\text {MAX }}$ values to determine the area number density. For calculation of both $V_{\text {MAX }}$ and $A_{\text {MAX }}$, the detector area used is roughly $85 \%$ of the total area of the WFC3 IR chip (4.5 $\left.\operatorname{arcmin}^{2}\right)$, as objects are lost to the edges where either the first-order spectrum falls off the chip (right side) or there are no direct images of galaxies available (left side).

The primary surveys of the future near-infrared grism space missions, Euclid (Laureijs et al. 2012) and WFIRST (Green et al. 2012; Dressler et al. 2012), will both cover thousands of square degrees to depths significantly shallower than the WISP program. The Euclid wide spectroscopic survey will cover $15,000 \mathrm{deg}^{2}$ over a wavelength range of $1.1-2 \mu \mathrm{m}$, with a resolution of $R=250$ and a $3.5 \sigma$ line flux depth of $3 \times 10^{-16} \mathrm{erg} \mathrm{s}^{-1} \mathrm{~cm}^{-2}$. At time of publication, the WFIRST
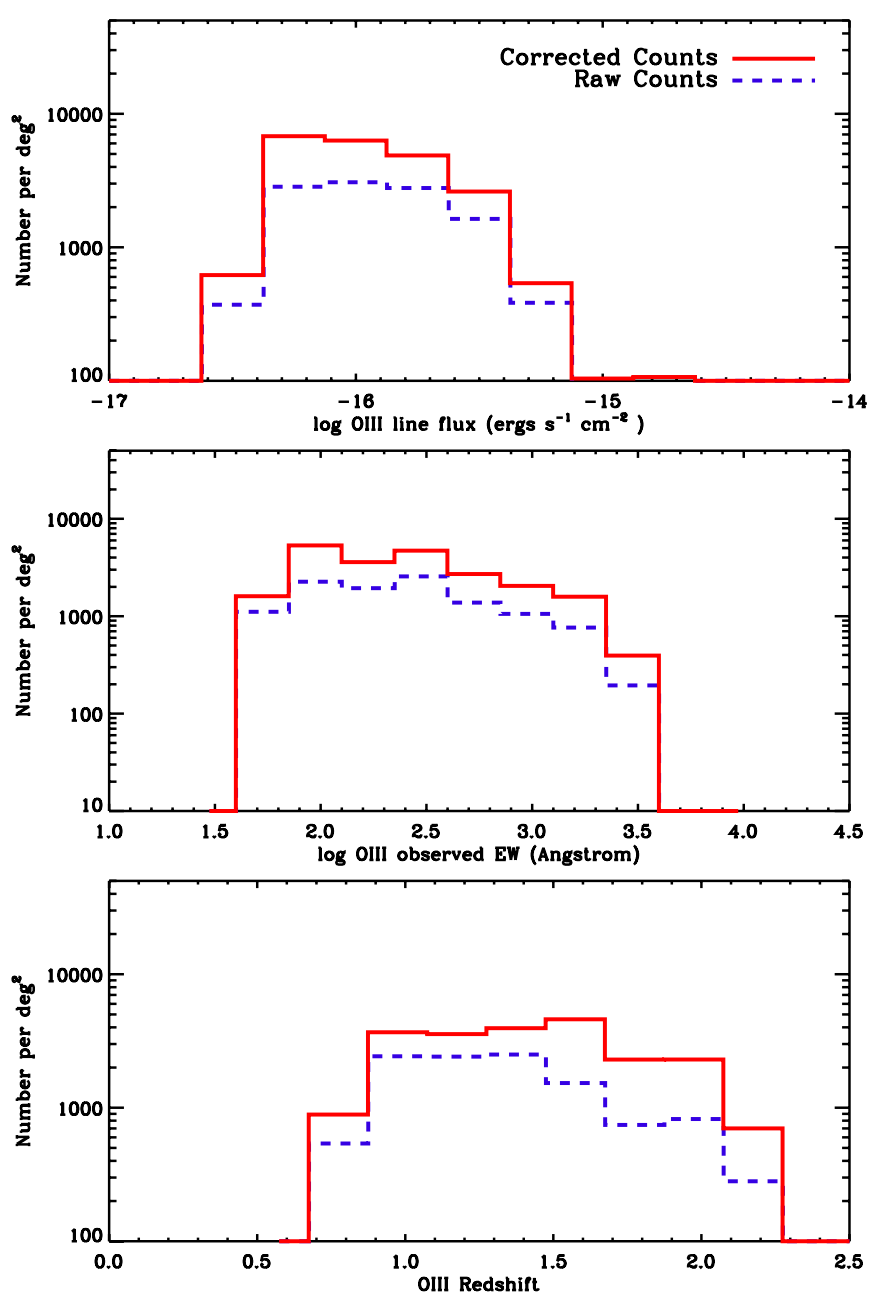

Figure 7. Histogram of all detected [O III] line emitters as a function of (top) line flux, (middle) observed equivalent width, and (bottom) redshift. The dashed blue lines are the raw, uncorrected counts, while the solid red lines are the counts after the correction for completeness. We note that the faintest [O III] flux bins consist mostly of secondary, fainter lines that have only been identified and included because they are companions to brighter $\mathrm{H} \alpha$ emission lines. We make no attempt to correct for this incompleteness, choosing instead to restrict our analysis to the brighter lines.

(A color version of this figure is available in the online journal.)

mission is still considering several competing designs, but the primary spectroscopic mission will likely cover at least $2000 \mathrm{deg}^{2}$ at a resolution of $R=600$, down to a $7 \sigma$ line flux depth of $1 \times 10^{-16} \mathrm{erg} \mathrm{s}^{-1} \mathrm{~cm}^{-2}$. The wavelength range for WFIRST will likely start between 1.3 and $1.5 \mu \mathrm{m}$ and end somewhere between 2 and $2.4 \mu \mathrm{m}$. While this paper focuses on comparisons and predictions for the future shallow wide surveys of Euclid and WFIRST, it should be noted that both missions will certainly contain deeper surveys done over less area. For instance, the Euclid mission plan contains at least two $20 \mathrm{deg}^{2}$ deep fields with planned depths very similar to the observations for the WISP program, with line flux limits around $5 \times 10^{-17} \mathrm{erg} \mathrm{s}^{-1} \mathrm{~cm}^{-2}$.

In Figure 8, we plot cumulative number counts versus limiting flux for the WISP survey, covering the primary range of interest $\left((1-10) \times 10^{-16} \mathrm{erg} \mathrm{s}^{-1} \mathrm{~cm}^{-2}\right)$ of these future surveys. Completeness corrections have been applied to all points, and the plotted errors include those derived from our simulations of the completeness rates (see Figure 4) combined with the Poissonian error. There are no corrections to the fluxes for extinction or the contribution from $[\mathrm{N} I \mathrm{I}]$, which is completely 


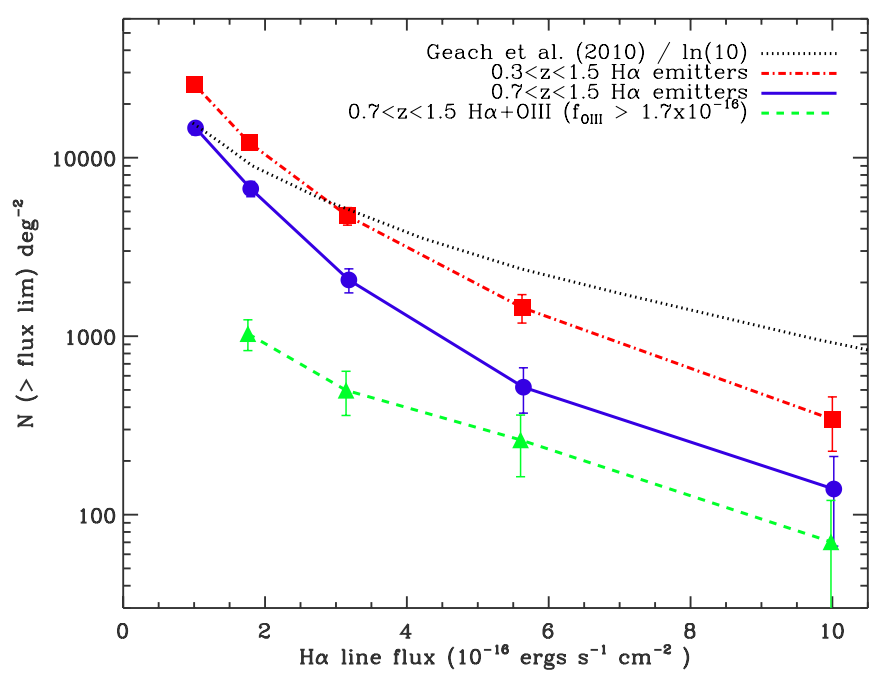

Figure 8. Cumulative number count density as a function of $\mathrm{H} \alpha$ line flux. The red squares and dot-dashed line are all the $0.3<z<1.5 \mathrm{H} \alpha$ emitters, the blue circles and solid line are just $\mathrm{H} \alpha$ emitters between $0.7<z<1.5$, while the green triangles and dashed line are $\mathrm{H} \alpha$ emitters with [O III] line emission greater than $1.7 \times 10^{-16} \mathrm{erg} \mathrm{s}^{-1} \mathrm{~cm}^{-2}$ (approximately $2 \sigma$ for the planned Euclid line flux limits). The dotted black line is the number count density prediction of Geach et al. (2010), divided by $\ln (10)$. Please note that no corrections for dust extinction or [N II] contamination have been applied to any of the fluxes plotted for cumulative counts in Figures 8-10.

(A color version of this figure is available in the online journal.)

blended with $\mathrm{H} \alpha$ for WISP. We present three cumulative number measurements: total $\mathrm{H} \alpha$ emitters (red squares), $0.7<z<1.5$ $\mathrm{H} \alpha$ emitters (blue circles), and $0.7<z<1.5 \mathrm{H} \alpha$ emitters for which [O III] is also detected (green triangles). At the bright flux end $\left(10^{-15} \mathrm{erg} \mathrm{s}^{-1} \mathrm{~cm}^{-2}\right)$ we find a factor of 1.5 times more $\mathrm{H} \alpha$ emission lines at $0.3<z<0.7$ than at $0.7<z<1.5$. As we approach fainter fluxes, the percentage of high-redshift objects increases, with the number of $0.7<z<1.5$ sources exceeding those at $0.3<z<0.7$ around $2 \times 10^{-16} \mathrm{erg} \mathrm{s}^{-1} \mathrm{~cm}^{-2}$.

The WISP survey reaches shorter wavelengths $(0.85 \mu \mathrm{m})$ than currently planned for these future space missions. The Euclid short wavelength limit, $1.1 \mu \mathrm{m}$, translates into an $\mathrm{H} \alpha$ redshift of $z=0.7$, so we use the $0.7<z<1.5$ cumulative number counts to predict what these future space missions will find, noting that the WFIRST number counts will be somewhat lower depending on the exact low wavelength cut-off used. The $1.65 \mu \mathrm{m}$ WISP cut-off is a shorter long-wavelength cut-off than the $2 \mu \mathrm{m}$ or better planned for these future missions, but that only means the WISP survey is not sensitive to the highest redshift $\mathrm{H} \alpha$ emitters. Because of the steep fall-off in number counts with redshift for the flux limits being planned, it is unlikely that the cumulative $\mathrm{H} \alpha$ number counts presented will be much different even with longer wavelength sensitivity.

We find approximately $2500(18,500) \mathrm{H} \alpha$ emitters $\mathrm{deg}^{-2}$ in the redshift range $0.7<z<1.5$ down to 3 (1) $\times 10^{-16} \mathrm{erg} \mathrm{s}^{-1} \mathrm{~cm}^{-2}$. For comparison, we plot the $0.75<$ $z<1.9$ number counts predicted by the study of Geach et al. (2010), which is mostly based on HST NICMOS grism and near-IR narrowband number counts (Shim et al. 2009; Geach et al. 2008; Sobral et al. 2009). We have decreased the result from the published article by a factor of $\ln (10)$ to account for an error in the published values of $\Phi_{\star}$ used, a result of improper conversion of $\Phi(\log L)$ luminosity functions to the more standard $\Phi(L)$ luminosity functions. We have also shifted the Geach et al. (2010) predicted counts to brighter fluxes by a factor of
1.4 to account for the $[\mathrm{N} \mathrm{II}]$ contamination that is included in the WISP counts but has been removed from the counts of Geach et al. (2010). Once we account for these offsets, we find that the NICMOS-derived cumulative distribution and the WISP distribution approach each other at the faint end, but that there is an increasingly large disagreement toward the brighter fluxes, reaching a factor of six difference by $10^{-15} \mathrm{erg} \mathrm{s}^{-1} \mathrm{~cm}^{-2}$. This large disparity at the bright flux end is likely a result of the NICMOS luminosity functions (Yan et al. 1999; Shim et al. 2009) used to normalize the Geach et al. (2010) number count models. The NICMOS luminosity functions contain significantly more high-luminosity (>3 $>10^{42} \mathrm{erg} \mathrm{s}^{-1}$ ) $\mathrm{H} \alpha$ line emitters than we see for WISP (see Section 4.3).

We are in better agreement with the emission-line galaxy count predictions of Wang et al. (2013), who also find a number density of roughly $1000 \mathrm{deg}^{-2}$ down to an $\mathrm{H} \alpha$ flux of $4 \times 10^{-16} \mathrm{erg} \mathrm{s}^{-1} \mathrm{~cm}^{-2}$. Note that this flux limit is higher than the approximately $3 \times 10^{-16} \mathrm{erg} \mathrm{s}^{-1} \mathrm{~cm}^{-2}$ flux limit given in Wang et al. (2013), but to properly compare the two we had to add the [N II] contribution back into their $\mathrm{H} \alpha$ fluxes. The Wang et al. (2013) predictions are based on the $\mathrm{H} \alpha$ luminosity functions from the narrowband survey of Sobral et al. (2013), with which we also largely agree (see Section 4.3 below). The general agreement between these two different emission line detection methodologies (near-infrared grism and narrowband filters) suggests that the counts presented here are a robust measurement of the number of $\mathrm{H} \alpha$-emitting galaxies.

To predict the number of multiple emission line $(\mathrm{H} \alpha+$ [O III]) sources a future near-infrared slitless spectroscopy space mission could expect to find per square degree, one must first choose a flux limit down to which [O III] will also be detectable. This detection limit will vary depending on survey depth and secondary line reliability requirements. We can start with the currently planned Euclid mission $3.5 \sigma$ detection limit of $3 \times 10^{-16} \mathrm{erg} \mathrm{s}^{-1} \mathrm{~cm}^{-2}$. If we make the further assumption that one could reach a lower significance limit of $2 \sigma$ for a secondary line if one already had a higher confidence line in hand, then one could detect [O III] flux down to $1.7 \times 10^{-16} \mathrm{erg} \mathrm{s}^{-1} \mathrm{~cm}^{-2}$. We apply this [O III] flux limit to the green cumulative count line in Figure 8, producing a prediction for Euclid for the number density of emission-line galaxies where both $\mathrm{H} \alpha$ and [O III] will be detected. Down to an $\mathrm{H} \alpha$ flux of $3 \times 10^{-16} \mathrm{erg} \mathrm{s}^{-1} \mathrm{~cm}^{-2}$ we find that roughly $24 \%$ of the $0.7<z<1.5 \mathrm{H} \alpha$ emitters are also detected in [O III], or $600 \mathrm{H} \alpha+[\mathrm{O} \mathrm{III}]$ emitters per square degree. A higher limit on the [O III] flux will obviously exclude more multiple emission line objects from the final counts. For instance, if we require the [O III] and $\mathrm{H} \alpha$ line fluxes to both be greater than $3 \times 10^{-16} \mathrm{erg} \mathrm{s}^{-1} \mathrm{~cm}^{-2}$, then the density of $\mathrm{H} \alpha+$ [O III] emitters drops by half, down to around $280 \mathrm{deg}^{-2}$.

With 314 detected (before completeness correction) $\mathrm{H} \alpha$ emission lines at $z>0.7$ with fluxes above $10^{-16} \mathrm{erg} \mathrm{s}^{-1} \mathrm{~cm}^{-2}$, we have enough emission lines to break down the cumulative number counts into even smaller redshift bins in Figure 9. This more detailed redshift breakdown allows us to examine the evolution of the number counts that will be available to these future near-infrared slitless spectrographs in space. At a flux of $10^{-15} \mathrm{erg} \mathrm{s}^{-1} \mathrm{~cm}^{-2}$ the $0.7<z<1.5 \mathrm{H} \alpha$ emission line galaxies are split roughly equally between $0.7<z<0.95$ and $0.95<$ $z<1.2$. However, by $6 \times 10^{-16} \mathrm{erg} \mathrm{s}^{-1} \mathrm{~cm}^{-2}$, the emitters from the $0.95<z<1.2$ redshift range outnumber those from $0.7<z<0.95$ by a factor of $1.5-2$ and remain more numerous down to fainter fluxes. Our highest redshift bin, $1.2<z<1.5$, has no emission lines as bright as $10^{-15} \mathrm{erg} \mathrm{s}^{-1} \mathrm{~cm}^{-2}$ and is not 


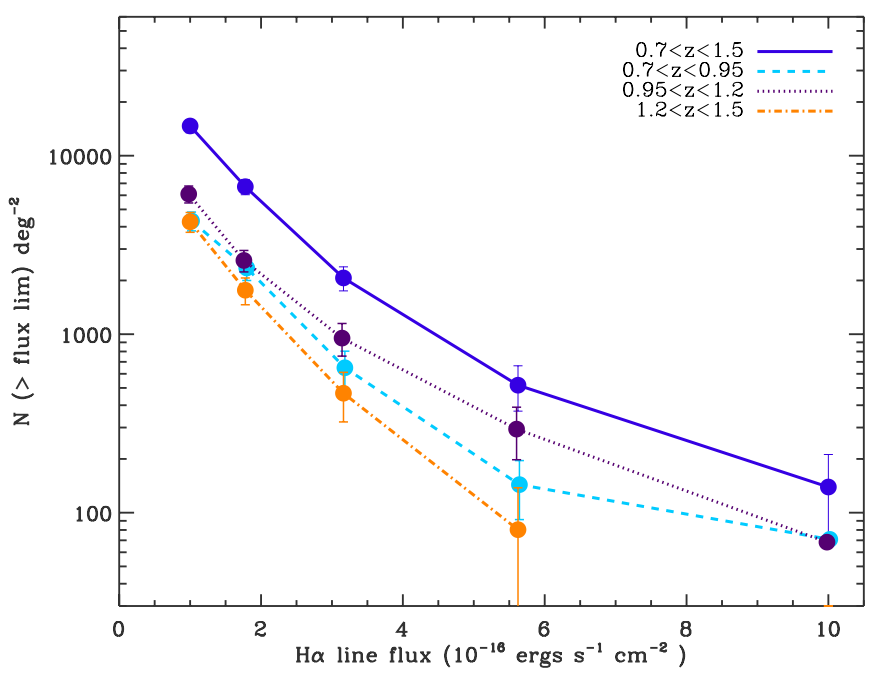

Figure 9. Cumulative number count density as a function of $\mathrm{H} \alpha$ line flux split into different redshift bins from $0.7<z<1.5$. The solid blue line is all emitters from $0.7<z<1.5$ and is unchanged from Figure 8 . The dashed light blue line is $0.7<z<0.95$, the dotted purple line is $0.95<z<1.2$, and the dot-dashed orange line is $1.2<z<1.5$.

(A color version of this figure is available in the online journal.)

a significant percentage of the emission-line sample until fluxes less than $\sim 3 \times 10^{-16} \mathrm{erg} \mathrm{s}^{-1} \mathrm{~cm}^{-2}$.

We can also look at the number densities of the [O III] emission lines on their own in Figure 10. Over the same range of line fluxes, the number counts of [O III] emitters initially rise much more slowly than the numbers of $\mathrm{H} \alpha$ emitters, before rising quickly for fluxes fainter than $3 \times 10^{-16} \mathrm{erg} \mathrm{s}^{-1} \mathrm{~cm}^{-2}$. At $10^{-15} \mathrm{erg} \mathrm{s}^{-1} \mathrm{~cm}^{-2}$, there are three times more $\mathrm{H} \alpha$ than [O III] emission lines, but by $6 \times 10^{-16} \mathrm{erg} \mathrm{s}^{-1} \mathrm{~cm}^{-2}$ that number density difference has increased to a factor of seven. Then, thanks to the steep rise of the fainter [O III] emitters, this number density disparity rapidly shrinks and returns to a factor of three for line fluxes of $10^{-16} \mathrm{erg} \mathrm{s}^{-1} \mathrm{~cm}^{-2}$. This steep rise in the relative $[\mathrm{O} \mathrm{III}]$ number density can also be seen in the decreasing $\mathrm{H} \alpha /[\mathrm{O}$ III] ratio we measure for fainter line fluxes (see Section 4.2 below).

We find roughly 1.5 times as many $0.7<z<1.5$ [O III] emitters, where $\mathrm{H} \alpha$ can also be identified in the WISP data, as [O III] emitters found at the higher $1.5<z<2.3$ redshifts. This is after accounting for the extra incompleteness [O III] suffers at the higher redshift (see Section 3.1.1). In the actual raw counts we find three times as many $0.7<z<1.5$ [O III] emitters. This number difference between redshifts remains fairly constant over the (1-5) $\times 10^{-16} \mathrm{erg} \mathrm{s}^{-1} \mathrm{~cm}^{-2}$ flux range. In the data we see an unexpected crossover toward the brightest end, with the highest redshift emitters becoming the most populous. However, the number statistics here are quite poor, with only nine total [O III] emitters covering the $(5-10) \times 10^{-16} \mathrm{erg} \mathrm{s}^{-1} \mathrm{~cm}^{-2}$ flux range, split into the two redshift bins. Within the error bars, we find that the number counts are consistent with no significant change in the number ratio between the high- and low-redshift [O III] emitters over the entire flux range. This relatively steady number count ratio across a wide range of redshifts suggests that the luminosity of the typical [O III] emitter $\left(L_{\star}\right)$ is increasing strongly with redshift, something we examine further when we look at the [O III] luminosity function in Section 4.3. All the cumulative count number predictions from Figures 8-10 are also listed in Table 2.

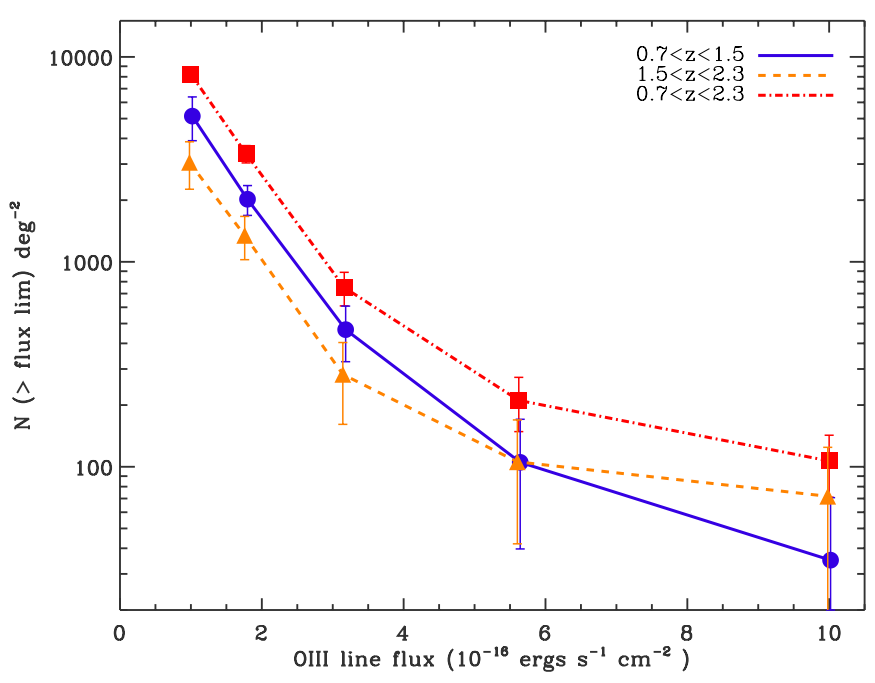

Figure 10. Cumulative number count density as a function of [O III] line flux, split into different redshift bins. The red squares and dot-dashed line are all emitters from $0.7<z<2.3$, the blue circles and solid line are the number density counts from $0.7<z<1.5$, while the orange triangles and dashed line are $1.5<z<2.3$

(A color version of this figure is available in the online journal.)

\section{2. $\mathrm{H \alpha} /[\mathrm{O}$ III $]$ Ratio}

Over the near-infrared wavelength range probed by the WISP survey, $0.85-1.65 \mu \mathrm{m}$, the vast majority of the emission lines observed are either $\mathrm{H} \alpha$ or [O III]. When other emission lines are identified, they are always found at the same time as one of those two lines, largely because these two emission lines are almost always significantly stronger than the other available lines. Besides the possibility of a rare [O II] emitter (see Section 3.3 above), our simulations suggest that the assumption that all single-line emitters are $\mathrm{H} \alpha$ mainly results in the misidentification of [O III] emission. While these failed identifications only affect $6 \%$ of $\mathrm{H} \alpha$ lines, it has a large effect on the high-redshift $(z>1.5)$ [O III] emitters, where we lose nearly $50 \%$ from our final sample.

The future near-infrared grism space missions are likely to suffer similar single emission line misidentification issues. Increased wavelength resolution will certainly aid in identification, as a resolved [O III] $\lambda \lambda 5007+4959$ doublet will not be confused with $\mathrm{H} \alpha$. However, for grism spectroscopy wavelength resolution is not the only limiting factor. If the emitting region is large in spatial extent, it will smear out the doublet, effectively lowering the available wavelength resolution. The median effective radius (the radius within which one-half of the total flux is contained, measured using the F110W filter) of our sample is only 0 '3. Using a similar set of HST near-infrared filters and grisms, van Dokkum et al. (2013) also found effective half-light radii of $\sim 0$ '.35 (3 kpc) across their sample of $z=0.5-2$ galaxies. However, future wide-area grism missions will reach shallower flux depths and therefore should find larger objects, as there is a strong correlation between line flux and galaxy size, at least for $\mathrm{H} \alpha$.

In Figure 11 we plot line flux versus effective radius for both $\mathrm{H} \alpha$ and [O III] emission line galaxies. The large squares (triangles) with error bars are the mean effective radii for each $\mathrm{H} \alpha$ ([O III]) flux bin, where each input emission-line galaxy has been weighted by the inverse of the completeness determined for size from Figure 1. Effective radius for $\mathrm{H} \alpha$ emitters shows a clear trend with line flux, $r_{\text {eff }}=3.6( \pm 1.5)$ 
Table 2

Emission-line Cumulative Counts ${ }^{\mathrm{a}}$

\begin{tabular}{|c|c|c|c|c|c|c|c|c|}
\hline Line Flux ${ }^{b}$ & $\begin{array}{c}\mathrm{H} \alpha \\
0.3<z<1.5\end{array}$ & $\begin{array}{c}\mathrm{H} \alpha \\
0.7<z<1.5\end{array}$ & $\begin{array}{c}\mathrm{H} \alpha \\
0.7<z<0.95\end{array}$ & $\begin{array}{c}\mathrm{H} \alpha \\
0.95<z<1.2\end{array}$ & $\begin{array}{c}\mathrm{H} \alpha \\
1.2<z<1.5\end{array}$ & $\begin{array}{c}\mathrm{H} \alpha+[\mathrm{O} \text { III }]^{\mathrm{c}} \\
0.7<z<1.5\end{array}$ & $\begin{array}{c}{[\mathrm{O} \text { III }]} \\
0.7<z<1.5\end{array}$ & $\begin{array}{c}{[\mathrm{O} \text { III }]} \\
1.5<z<2.3 \\
\end{array}$ \\
\hline 1.0 & 25,806 & 14,673 & 4311 & 6098 & 4263 & $\mathrm{n} / \mathrm{a}$ & 8241 & 3056 \\
\hline 1.8 & 12,127 & 6703 & 2350 & 2589 & 1764 & 1033 & 3366 & 1345 \\
\hline 3.2 & 4721 & 2066 & 649 & 951 & 467 & 498 & 749 & 282 \\
\hline 5.6 & 1447 & 517 & 144 & 294 & 80 & 262 & 211 & 106 \\
\hline 10.0 & 342 & 139 & 71 & 68 & 0 & 70 & 107 & 72 \\
\hline
\end{tabular}

Notes.

a All counts are per $\mathrm{deg}^{2}$. Fluxes have not been corrected for [N II] contamination.

b $10^{-16} \mathrm{erg} \mathrm{s}^{-1} \mathrm{~cm}^{-2}$

${ }^{c}$ Both $\mathrm{H} \alpha$ and $[\mathrm{O} \mathrm{III}]$ required to be greater than $1.7 \times 10^{-16} \mathrm{erg} \mathrm{s}^{-1} \mathrm{~cm}^{-2}$.

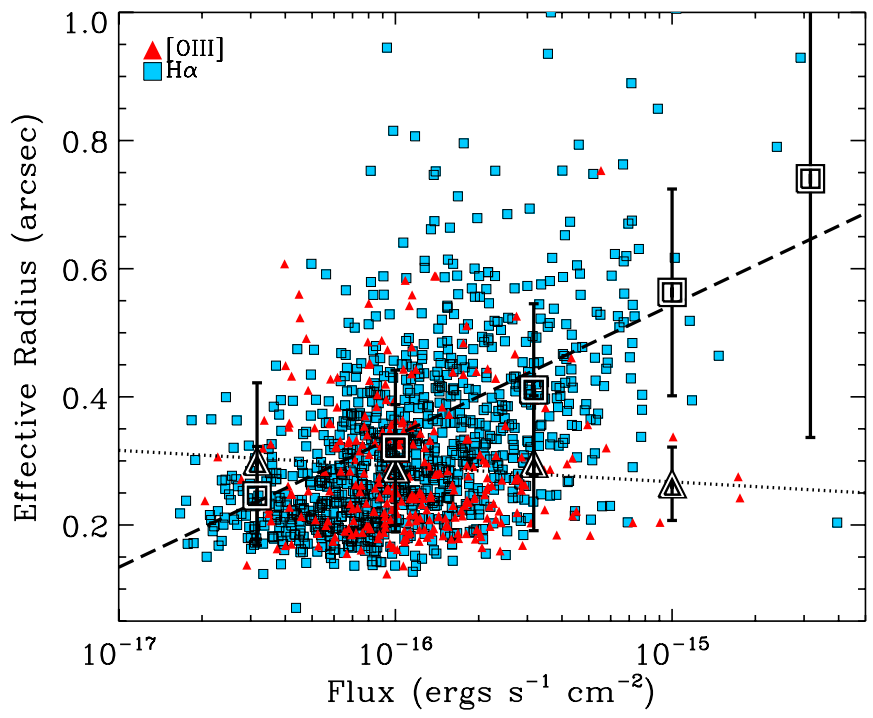

Figure 11. Flux vs. effective radius for our entire sample of line emitters, $\mathrm{EW}>40 \AA$ and $\mathrm{S} / \mathrm{N}>5$. H $\alpha$ and [O III] emitters are plotted as blue squares and red triangles, respectively. The large squares and triangles with error bars are the mean effective radii for each $\mathrm{H} \alpha$ or [O III] flux bin. The dashed $(\mathrm{H} \alpha)$ and dotted ([O III]) lines are the best linear fits to these mean effective radius bins.

(A color version of this figure is available in the online journal.)

$+0.2( \pm 0.1) \times \log F$, where the units of $r_{\mathrm{eff}}$ are arcseconds and $\log F$ is measured in erg s $\mathrm{s}^{-1} \mathrm{~cm}^{-2}$. However, there is virtually no trend for [O III] emitters at all, with our best fit being $r_{\text {eff }}=-0.1( \pm 1.1)-0.025( \pm 0.07) \times \log F$, essentially consistent with a constant effective radius over this range of [O III] emission line fluxes.

Down to $3 \times 10^{-16} \mathrm{erg} \mathrm{s}^{-1} \mathrm{~cm}^{-2}$ and limiting our analysis to redshifts of $z>1.2$ (where $\mathrm{H} \alpha /[\mathrm{O}$ III] confusion becomes an issue at $1.1 \mu \mathrm{m}$ ), we find that the radius of the median object increases $33 \%$ to 0. . 4 . While the sample of objects with both high fluxes and high redshifts is small, $13 \%$ ( 2 of 15 ) have radii larger than 0.6. Fortunately, the pixel scale for the Euclid mission will be 0.3 pixel $^{-1}$, more than twice that of the IR WFC3, which should greatly mitigate the degradation of wavelength resolution caused by extended sources. While the exact pixel scale for WFIRST detectors remains uncertain, it will likely be close to that of the WFC3 IR channel, 0 .'13 pixel $^{-1}$. However, the proposed WFIRST wavelength resolution $(R=600)$ is large enough that the wavelength smearing should not prevent identification of the $[\mathrm{O}$ III] $\lambda \lambda 5007+4959$ doublet in most cases.

To investigate the likelihood of single emission line confusion further, we plot the ratio of $\mathrm{H} \alpha /\left[\mathrm{O}_{\mathrm{III}}\right]$ as a function of $\mathrm{H} \alpha$ flux

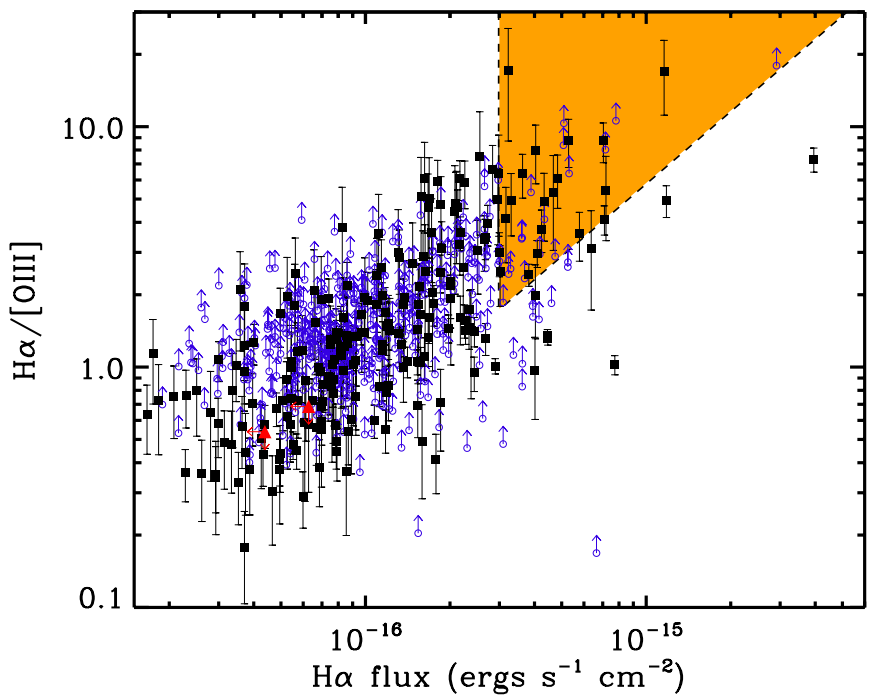

Figure 12. Ratio of $\mathrm{H} \alpha$ divided by [O III] line flux vs. $\mathrm{H} \alpha$ flux for all detected $0.7<z<1.5$ line emitters. Solid black squares are sources in which both lines are detected. The empty blue circles are sources where only $\mathrm{H} \alpha$ is detected and are lower limits, while the red triangles are the two sources where only [O III] has been detected, with its two arrows pointing toward the quadrant where those points must really lie. The orange wedge represents the area where one would expect to find only a single $\mathrm{H} \alpha$ emission line, assuming an $\mathrm{H} \alpha$ detection limit of $3 \times 10^{-16} \mathrm{erg} \mathrm{s}^{-1} \mathrm{~cm}^{-2}$ (planned Euclid survey limit) and a fainter [O III] detection limit of $1.7 \times 10^{-16} \mathrm{erg} \mathrm{s}^{-1} \mathrm{~cm}^{-2}$.

(A color version of this figure is available in the online journal.)

in Figure 12. We limit our redshift range to that for which both $\mathrm{H} \alpha$ and $\left[\mathrm{O}_{\mathrm{III}}\right]$ can be detected. Upper limits are $2 \sigma$, assuming that the FWHM of the line for which a limit is being derived is the same as for the measured emission line. We find only two cases where [O III] is detected without $\mathrm{H} \alpha$. We are able to identify these two emission lines as [O III] without secondary emission lines because they are compact and high enough $\mathrm{S} / \mathrm{N}$ that the distinctive [O III] doublet line profile is visible.

The upper left part of Figure 12, where the $\mathrm{H} \alpha /[\mathrm{O}$ III $]$ ratio is large and $\mathrm{H} \alpha$ fluxes are small, is empty of sources because that is where the flux limits of the survey prevent sampling. If the high $\mathrm{H} \alpha /[\mathrm{O} \mathrm{III}]$ ratios we see at brighter fluxes continue down to fainter fluxes, then presumably we would find many of the lower limits moving into that region if we were sensitive enough to detect such faint [O III] fluxes. The lower right portion of Figure 12, where $\mathrm{H} \alpha /[\mathrm{O} \mathrm{III}]$ ratio is small and the $\mathrm{H} \alpha$ fluxes are bright, is similarly devoid of objects, but that region is not excluded by our sensitivity limits. There is a real scarcity of low $\mathrm{H} \alpha /[\mathrm{O} \mathrm{III}]$ sources at the bright flux limits. Excluding three lower limits, we find that none of the 58 galaxies with 
$\mathrm{H} \alpha$ fluxes greater than $3 \times 10^{-16} \mathrm{erg} \mathrm{s}^{-1} \mathrm{~cm}^{-2}$ have ratios of $\mathrm{H} \alpha /[\mathrm{O}$ III $]<0.95$.

While the WISP survey does not cover the same wavelength range as the future space near-infrared grism missions (WISP goes to shorter wavelengths while the future missions will go longer), it does give us a rough estimate of the percentage of single line emitters those missions are likely to find. The exact percentage of single line emitters will depend on their final flux limits and criteria for establishing the reality of an emission line. For this example we again choose the currently planned detection limit for Euclid (3.5 $\sigma$ limit of $3 \times 10^{-16} \mathrm{erg} \mathrm{s}^{-1} \mathrm{~cm}^{-2}$ ) and assume that a secondary line only needs to be detected at a $2 \sigma$ significance $\left(1.7 \times 10^{-16} \mathrm{erg} \mathrm{s}^{-1} \mathrm{~cm}^{-2}\right)$. The shaded orange region in Figure 12 shows the region for which only single line emitters would be expected for these sets of limits. We find that at least $60 \%(35 / 58)$ of sources with emission lines brighter than $3 \times 10^{-16} \mathrm{erg} \mathrm{s}^{-1} \mathrm{~cm}^{-2}$ will be single line emitters in future surveys. This single-line discovery rate could potentially be as high as $80 \%$ if all the lower limits presented actually lie within the single-line discovery region.

We can also look at a sample where the redshift is $z>1.2$, as that would better reflect the range over which [O III] would be detectable if the lower wavelength cut-off is the $1.1 \mu \mathrm{m}$ cut-off of Euclid rather than the $0.85 \mu \mathrm{m}$ of the WISP survey. The number of bright emitters is much smaller (12), but the percentage of expected single line emitters, $67 \%-83 \%$, is virtually the same as when we included the lower redshifts emitters.

Our analysis assumes that all the single emission lines are $\mathrm{H} \alpha$, which we know to be incorrect roughly $10 \%$ of the time. Therefore, it is possible that approximately 3 of the 31 single line emitters in that flux range could be low $\mathrm{H} \alpha /[\mathrm{O}$ III] sources, although if their true redshifts are greater than $z=1.5$, then their $\mathrm{H} \alpha$ fluxes and $\mathrm{H} \alpha /[\mathrm{O} \mathrm{III}]$ ratios are not constrained. It is important to emphasize that of the 25 sources with bright fluxes where we detect both $\mathrm{H} \alpha$ and [O III], none of them have $\mathrm{H} \alpha$ / $\left[\mathrm{O}_{\mathrm{III}}\right]<1$. There is no obvious reason why we would be missing all the galaxies with low $\mathrm{H} \alpha /[\mathrm{O}$ III] from $0.7<z<1.5$, unless the ratio is so low that we lose the ability to detect $\mathrm{H} \alpha$. However, that would require an unlikely large gap in the $\mathrm{H} \alpha /[\mathrm{O} \mathrm{III}]$ ratio, making all galaxies either strong $\mathrm{H} \alpha$ or strong [O III] emitters, with none in between.

Regardless of the exact quantity of contamination from single-line $[\mathrm{O} \mathrm{III}]$ emission, clearly $\mathrm{H} \alpha /[\mathrm{O} \mathrm{III}]<1$ sources are much rarer at the bright fluxes that future near-infrared grism missions will probe than in our fainter sample. Therefore, the assumption that all single-line emitters are $\mathrm{H} \alpha$ should produce very few contaminating interlopers for these space missions, although further data are required to better establish exactly what the [O III] contamination rate is.

The trend of increasing $\mathrm{H} \alpha /[\mathrm{O} \mathrm{III}]$ ratio with $\mathrm{H} \alpha$ flux is a result of the known correlation between the $\mathrm{H} \alpha /[\mathrm{O}$ III] ratio and $\mathrm{H} \alpha$ luminosity previously reported by WISP (Domínguez et al. 2013). Their analysis also indicates that dust extinction increases with $\mathrm{H} \alpha$ luminosity, meaning that dust extinction is suppressing [O III] emission more at the bright luminosity end. However, the trend of stronger relative [O III] with fainter $\mathrm{H} \alpha$ luminosity is far stronger than their measured effects of extinction. The dominant determinant of the $\mathrm{H} \alpha /[\mathrm{O} \mathrm{III}]$ ratio is most likely the metallicity, as the $[\mathrm{O} \mathrm{III}] \lambda 5007 / \mathrm{H} \beta$ ratio strongly correlates with metallicity in the local universe (i.e., Liang et al. 2006). Metallicity is known to correlate with mass and luminosity (Tremonti et al. 2004; Kobulnicky \& Kewley 2004), so it is not surprising that the brightest $\mathrm{H} \alpha$ emitters, which tend to be the most luminous

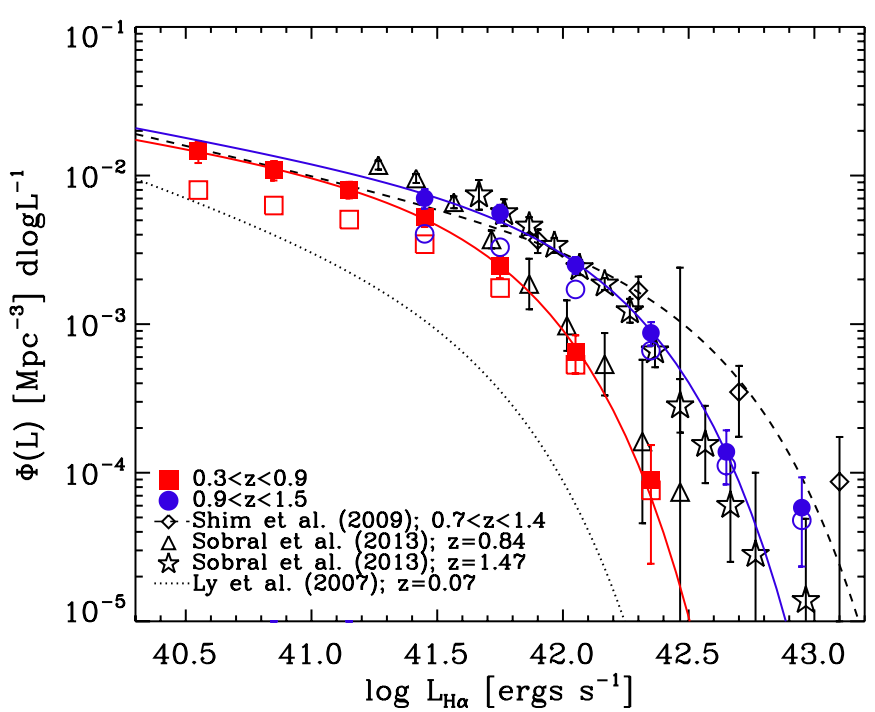

Figure 13. H $\alpha$ luminosity function derived from WISP emission-line sample. The red squares are line emitters from $0.3<z<0.9$, while the blue circles are line emitters from $0.9<z<1.5$. Best-fit Schechter functions have been overlaid on top with solid blue and red lines. The empty circles and squares are raw counts, while the solid circles and squares have been corrected for completeness. The diamonds and dashed line are the measured points and Schechter fit from the NICMOS grism study of Shim et al. (2009) for $0.7<z<1.4$. The triangles and stars are from the HiZELS narrowband study (Sobral et al. 2013) at $z=0.84$ and $z=1.47$, respectively. The dotted line is the $z=0.07 \mathrm{H} \alpha$ luminosity function (Ly et al. 2007).

(A color version of this figure is available in the online journal.)

in the continuum as well, would also have higher $\mathrm{H} \alpha /[\mathrm{O}$ III] ratios.

Another possible reason for the enhancement of [O III] could be the presence of an active galactic nucleus (AGN), although its effect on the $\mathrm{H} \alpha /[\mathrm{O} \mathrm{III}]$ ratio would be at least somewhat mitigated by the similar enhancement of [N II], which cannot be disentangled from $\mathrm{H} \alpha$ at the WISP wavelength resolution. However, line ratios from WISP stacked spectra are more consistent with the low-metallicity tail of a star-forming region than that of an AGN (Domínguez et al. 2013). This agrees with Garn et al. (2010), who found that $z=0.84 \mathrm{H} \alpha$-selected samples contain only 5\%-11\% AGNs. Sobral et al. (2013) suggest the AGN fraction does increase with redshift, but remains only $15 \%$ of the $\mathrm{H} \alpha$ emitters above $z>1$, with the biggest increases in AGN contribution at the brightest luminosities. While it is likely that some of the galaxies with the smallest $\mathrm{H} \alpha /[\mathrm{O}$ III] ratios are AGNs, they do not appear to make up enough of the total population of $\mathrm{H} \alpha$ emitters to have a significant effect on the overall trend of increasing $\mathrm{H} \alpha /[\mathrm{O}$ III] ratio with $\mathrm{H} \alpha$ flux.

\subsection{Luminosity Functions}

Using the total volume densities derived for each luminosity bin (see Section 4.1), we are able to plot $\mathrm{H} \alpha$ luminosity functions in Figure 13 and Table 3. We have split the $\mathrm{H} \alpha$ emission line luminosity function into two redshift ranges, $0.3<z<0.9$ and $0.9<z<1.5$. We chose to split the sample at $z=0.9$ as that divides the detected $\mathrm{H} \alpha$ emission lines by the grism with which they were detected (the dividing line between G102 and G141 is $\sim 1.2 \mu \mathrm{m}$ ), which also has the added benefit of producing roughly even sample sizes (436 $0.3<z<0.9$ versus 517 $0.9<z<1.5)$. We make no correction for dust extinction, but there is a correction factor of 0.71 applied to the luminosities to account for $[\mathrm{N}$ II $]$ contamination. Please note that before directly 
Table 3

$\mathrm{H} \alpha$ Luminosity Functions

\begin{tabular}{|c|c|c|c|c|c|c|}
\hline \multirow[t]{2}{*}{$\log L^{\mathrm{a}}$} & \multicolumn{3}{|c|}{$0.3<z<0.9$} & \multicolumn{3}{|c|}{$0.9<z<1.5$} \\
\hline & $\begin{array}{l}\text { Number } \\
\text { of Sources }\end{array}$ & $\begin{array}{c}\text { Density } \\
\left(\mathrm{Mpc}^{-3} d \log L^{-1}\right)\end{array}$ & $\begin{array}{l}\text { Corrected Density } \\
\left(\mathrm{Mpc}^{-3} d \log L^{-1}\right)\end{array}$ & $\begin{array}{l}\text { Number } \\
\text { of Sources }\end{array}$ & $\begin{array}{c}\text { Density } \\
\left(\mathrm{Mpc}^{-3} d \log L^{-1}\right)\end{array}$ & $\begin{array}{l}\text { Corrected Density } \\
\left(\mathrm{Mpc}^{-3} d \log L^{-1}\right)\end{array}$ \\
\hline 40.55 & 80 & 0.0080 & $0.0145 \pm 0.0024$ & $\mathrm{n} / \mathrm{a}$ & $\mathrm{n} / \mathrm{a}$ & $\mathrm{n} / \mathrm{a}$ \\
\hline 40.85 & 97 & 0.0063 & $0.0109 \pm 0.0016$ & $\mathrm{n} / \mathrm{a}$ & $\mathrm{n} / \mathrm{a}$ & $\mathrm{n} / \mathrm{a}$ \\
\hline 41.15 & 107 & 0.0051 & $0.0080 \pm 0.0010$ & 34 & 0.0087 & $0.0148 \pm 0.0036$ \\
\hline 41.45 & 90 & 0.0035 & $0.0052 \pm 0.0007$ & 143 & 0.0041 & $0.0070 \pm 0.0010$ \\
\hline 41.75 & 46 & 0.0017 & $0.0025 \pm 0.0004$ & 185 & 0.0033 & $0.0056 \pm 0.0008$ \\
\hline 42.05 & 14 & 0.0005 & $0.0007 \pm 0.0002$ & 103 & 0.0017 & $0.0025 \pm 0.0003$ \\
\hline 42.35 & 2 & $7.6 e-05$ & $8.9 \mathrm{e}-05 \pm 6.5 \mathrm{e}-05$ & 41 & 0.00066 & $0.00087 \pm 0.00016$ \\
\hline 42.65 & $\mathrm{n} / \mathrm{a}$ & $\mathrm{n} / \mathrm{a}$ & $\mathrm{n} / \mathrm{a}$ & 7 & 0.00011 & $0.00014 \pm 5.5 \mathrm{e}-05$ \\
\hline 42.95 & $\mathrm{n} / \mathrm{a}$ & $\mathrm{n} / \mathrm{a}$ & $\mathrm{n} / \mathrm{a}$ & 3 & $4.8 \mathrm{e}-05$ & $5.8 \mathrm{e}-05 \pm 3.5 \mathrm{e}-05$ \\
\hline 43.25 & $\mathrm{n} / \mathrm{a}$ & $\mathrm{n} / \mathrm{a}$ & $\mathrm{n} / \mathrm{a}$ & 1 & $1.6 \mathrm{e}-05$ & $1.6 \mathrm{e}-05 \pm 1.7 \mathrm{e}-05$ \\
\hline
\end{tabular}

Notes.

${ }^{\text {a }}$ Luminosity has been corrected for [N II] contamination. No extinction correction has been applied.

${ }^{\mathrm{b}}$ Corrected density is the measured number density with completeness corrections applied.

Table 4

Luminosity Function Best-fit Schechter Parameters

\begin{tabular}{lcccc}
\hline \hline Emission Line & Redshift Range & $\Phi_{\star}$ & $L_{\star}$ & $\alpha$ \\
\hline $\mathrm{H} \alpha$ & $0.3<z<0.9$ & $-2.51 \pm 0.11$ & $41.72 \pm 0.09$ & $-1.27 \pm 0.12$ \\
$\mathrm{H} \alpha$ & $0.9<z<1.5$ & $-2.70 \pm 0.12$ & $42.18 \pm 0.10$ & $-1.43 \pm 0.17$ \\
{$[\mathrm{O}$ III $]$} & $0.7<z<1.5$ & $-3.19 \pm 0.09$ & $42.34 \pm 0.06$ & $-1.40 \pm 0.15$ \\
{$[\mathrm{O}$ III $]$} & $1.5<z<2.3$ & $-3.74 \pm 0.43$ & $42.91 \pm 0.37$ & $-1.67 \pm 0.78$ \\
{$[\mathrm{O}$ III $]$} & $0.7<z<1.5$ & $-3.28 \pm 0.09$ & $42.39 \pm 0.08$ & -1.5 (fixed) \\
{$[\mathrm{O}$ III $]$} & $1.5<z<2.3$ & $-3.60 \pm 0.14$ & $42.83 \pm 0.11$ & -1.5 (fixed) \\
\hline
\end{tabular}

comparing these luminosity functions to the cumulative counts from Section 4.1, the [N II] contamination correction must be removed. Our best-fit Schechter parameters are presented in Table 4.

Generally we find that the $0.9<z<1.5$ luminosity function is comparable at the faint luminosity end to luminosity functions measured by the previous NICMOS grism studies (Yan et al. 1999; Shim et al. 2009). However, the WISP number densities drop more quickly as the $\mathrm{H} \alpha$ luminosities become brighter, dropping well below the bright luminosity number counts from previous NICMOS surveys by $\log L=42.5$. While many of the earlier NICMOS surveys are too small in area for accurate counts at the bright end of the luminosity function (Yan et al. 1999; Hopkins et al. 2000), the Shim et al. (2009) luminosity function plotted here is derived from an area roughly comparable $\left(104 \mathrm{arcmin}^{2}\right)$ to the WISP data presented in this work. With more than 139 different fields spread across the sky, the expected cosmological variance for Shim et al. (2009) is also low ( $<2 \%$; Trenti \& Stiavelli 2008), so it cannot explain the observed difference either. Finally, the redshift sampling difference $(0.7<z<1.4$ with NICMOS versus $0.9<z<1.5$ with WFC3) is not sufficiently different to effect the luminosity function comparison. The majority of deviation between the two luminosity functions comes down to a single bin at $\log L=42.7$, so the observed luminosity function difference may simply be a result of an unfortunate $2 \sigma-3 \sigma$ deviation at that single point.

We also find our $0.9<z<1.5$ number densities to be consistent with the $z=1.47$ number densities derived from the narrowband $\mathrm{H} \alpha$ surveys (Sobral et al. 2013). However, the redshifts sampled by Sobral et al. (2013) (at $z=0.84$ and 1.47) straddle the sampled range of our high-redshift sample (median redshift of $z=1.15$ ), so if all the luminosity functions were consistent, then the WISP luminosity function ought to lie between the two Sobral et al. (2013) distributions. That the number densities at $z=1.47$ and our median $z=1.15$ instead overlap suggests that the WISP grism survey may be finding slightly more line emitters than the narrowband survey, although the absolute differences are very small. Alternatively, the evolution in the luminosity function between $z=0.8$ and $z=1.5$ could be occurring mostly at the low end of that redshift range, as suggested by Geach et al. (2010). The HiZELS data sample down to very similar EWs $(25 \AA)$, so that is not a likely source of the difference. Neither data sample has made any attempt to remove AGNs, so that cannot be a source of difference either. There is some difference in the extraction apertures used. HiZELs uses a fixed $2^{\prime \prime}$ aperture at $z=1.47$, while WISP uses a varying aperture of twice the object size in the direction perpendicular to the wavelength dispersion (there is effectively no limit to the size of line emission detectable along the dispersion axis). However, the majority of objects are compact enough that it seems unlikely that either extraction methodology could be missing much flux.

Our lower redshift $0.3<z<0.9$ sample (median $z=$ 0.58), on the other hand, does fall below the Sobral et al. (2013) narrowband $z=0.84$ number counts, as well as the $z=0.8$ narrowband counts (not plotted) from the NEWFIRM $\mathrm{H} \alpha$ Survey (Ly et al. 2011), although the difference at the bright end of the luminosity function is within the errors. The more consistent low-redshift luminosity densities suggest that the WISP survey is not generally finding line emitters at a higher rate than narrowband surveys, but only for its higher redshift bin. A single minor inconsistency such as this could be partially explained by cosmological variance, which is expected to be 
on the order of $15 \%$ (Trenti \& Stiavelli 2008) for the $z=1.47$ Sobral et al. (2013) narrowband sample.

A significant strength of the WISP survey compared to narrowband studies is the relatively small contribution of cosmological variance to the overall error budget. First, the large difference in redshift depth $(\Delta z=1.2$ for WISP versus $\Delta z=0.02$ for a typical narrow band) means that despite covering a much smaller area of sky (e.g., $130 \operatorname{arcmin}^{2}$ versus 1-2 $\mathrm{deg}^{2}$ ), the WISP survey actually covers a comparable amount of volume. For instance, a single WISP pointing covers roughly $10^{4} \mathrm{Mpc}^{3}$ $(z=0.3-1.5)$, while the $z=0.84$ and $z=1.47$ narrowband samples of Sobral et al. (2013) cover $10^{4} \mathrm{Mpc}^{3}$ over 210 and 100 $\operatorname{arcmin}^{2}$, respectively. The 29 fields used in this paper survey a volume of nearly $3 \times 10^{5} \mathrm{Mpc}^{3}$, roughly equivalent to $1 \mathrm{deg}^{2}$ of the $z=1.47$ narrow band of Sobral et al. (2013).

Secondly, pencil-beam studies like WISP, which cover a small area over a large redshift range, have significantly lower cosmological variance than a similar cubical or spherical volume, as the long, narrow window must pass through many different environments while a regularly shaped cube could potentially lie right on top of an extreme overdensity (Trenti \& Stiavelli 2008). For objects with a density of $10^{-3} \mathrm{Mpc}^{-3}$, the cosmic variance is only $\sim 12 \%$ for a single WISP pencil beam, while it is nearly $60 \%$ for $z=0.85$ and $z=1.47$ narrow beam samples covering the same volume (Trenti \& Stiavelli 2008). Of course, the total areas covered by the Sobral et al. (2013) narrowband surveys are 6-20 times larger (1-2 deg ${ }^{2}$ ) from two separate fields, reducing their associated cosmological variance to $15 \%-20 \%$. However, as the WISP fields are widely separated on the sky, the cosmological variance from field to field is uncorrelated, reducing the cosmological variance of the total sample by the square root of the number of fields. For our example of $10^{-3}$ objects per $\mathrm{Mpc}^{3}$, the contribution of cosmological variance to the WISP sample presented in this paper is only $2 \%$. We have included cosmological variance in our calculations of total errors for the luminosity functions, although its contribution is essentially negligible compared to the Poissonian uncertainty and completeness correction errors.

We note that while a deep pencil-beam study like WISP has the advantage of very little cosmological variance because of the large redshift range observed, it does so by sacrificing the ability to sample the behavior of galaxies at more discrete redshifts. In effect, it blurs all the evolutionary effects that take place within each redshift bin. This averaging of galaxy evolution is not a significant issue if the evolution over the sampled redshift range is taking place at a steady, predictable pace, but could be misleading if the galaxy behavior changed particularly rapidly or became strongly non-linear. Of course, if the evolution becomes too rapid or unpredictable, narrowband surveys risk completely missing significant behavior that happens to fall between their sampled redshifts. Because of this, deep pencil-beam grism and narrowband surveys are actually complementary, each a check on the potential weaknesses of the other.

Regardless of how our sample selection may differ from other surveys, we can robustly compare how the luminosity function evolves within the WISP survey as we use the same methodology for selection and completeness corrections. It appears that most of the evolution from $z=0.3-1.5$ takes place at the bright end of the luminosity function. Within the errors, the luminosity functions are converging below $\log L=41$. However, with only two low-luminosity bins for the high-redshift sample, this lowluminosity convergence is not definitive. Generally it appears that most of the evolution in $\mathrm{H} \alpha$ from $z=0.3-1.5$ is taking

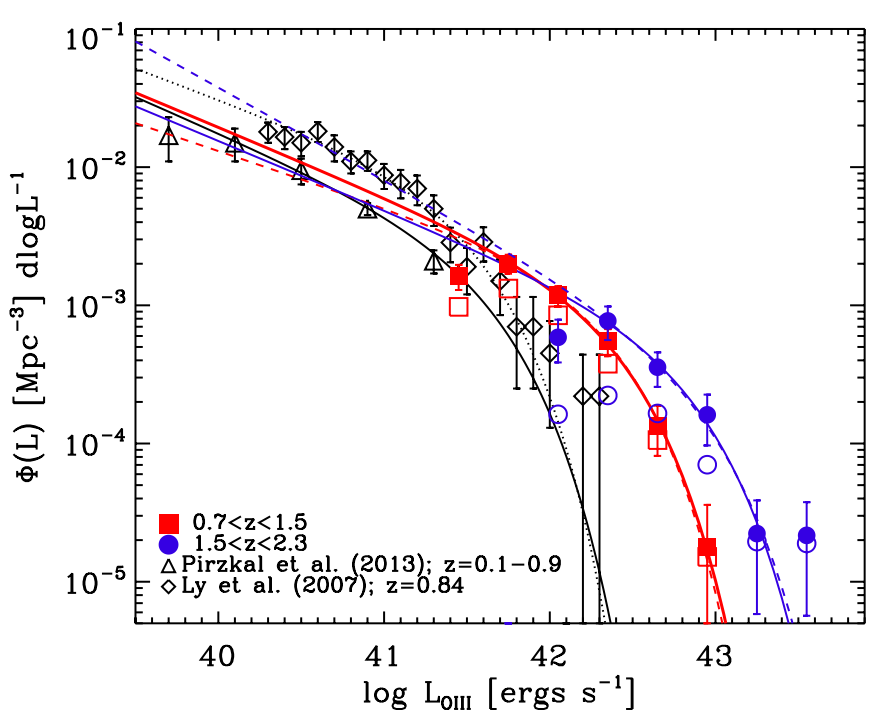

Figure 14. [O III] luminosity function derived from WISP emission-line sample. The red squares are $[\mathrm{O}$ III] emitters from $0.7<z<1.5$, while the blue circles are $1.5<z<2.3$. We plot Schechter function fits with both a fixed $\alpha=$ -1.5 (solid lines) and one where we allow $\alpha$ to vary (dashed lines). The empty circles and squares are raw counts, while the solid circles and squares have been corrected for completeness. The triangles and solid line fit are from the $z=$ 0.1-0.9 PEARS Grism Survey (Pirzkal et al. 2013), while the diamonds and dotted line fit are from the $z=0.84$ narrowband study of Ly et al. (2007).

(A color version of this figure is available in the online journal.)

place in $L_{\star}$, which is consistent with previous findings (Sobral et al. 2013). Further discussion of the $\mathrm{H} \alpha$ luminosity functions, their implied star formation rates, and the evolution of the star formation density will be presented in a separate paper (A. Bunker et al., in preparation).

We also present the [O III] luminosity functions in Figure 14 and Table 5 along with their accompanying best-fit Schechter parameters in Table 4. For [O III] we split luminosity functions into two redshift ranges, $0.7<z<1.5$ and $1.5<z<2.3$, which are the highest redshifts for which the [O III] luminosity function has been determined to date. The lower redshift bins cover the range over which the $\mathrm{H} \alpha$ line can also be found within the grism spectra. For $z>1.5, \mathrm{H} \alpha$ is redshifted beyond $1.65 \mu \mathrm{m}$, leaving [O III] as the most powerful detectable line in the grism spectrum. The total sample sizes are 192 [O III] emitters at $0.7<z<1.5$ versus 58 at $1.5<z<2.3$.

As remarked in Section 3.1.1, the [O III] number densities require higher completeness corrections than for $\mathrm{H} \alpha$. Even after application of these additional larger corrections, we still find that the lowest luminosity bin for each redshift range appears low, indicating that there is some incompleteness for which we are not accounting. One possibility is that the number of misidentified single-line [O III] emitters is even larger at the faintest ends than our simulations indicate. For instance, our simulations assume $\mathrm{H} \alpha /[\mathrm{O} \mathrm{III}]$ ratios similar to the emissionline sources that we find and can measure. Therefore, if a population of faint, low $\mathrm{H} \alpha /[\mathrm{O}$ III] sources exists, we would be underestimating the number of single-line misidentifications at the lower redshifts (at higher redshifts the $\mathrm{H} \alpha /\left[\mathrm{O}_{\mathrm{III}}\right]$ ratio does not matter as we cannot see $\mathrm{H} \alpha$ at all). Regardless of the reason for the low faintest bin, we exclude them from the Schechter fits and further analysis.

Comparing our [O III] luminosity functions to those derived at lower redshift (Pirzkal et al. 2013; Ly et al. 2007), we see a strong increase in $L_{\star}$ with redshift along with a decrease in $\Phi_{\star}$. 
Table 5

[O III] Luminosity Functions

\begin{tabular}{|c|c|c|c|c|c|c|}
\hline \multirow[t]{2}{*}{$\log L^{\mathrm{a}}$} & \multicolumn{3}{|c|}{$0.7<z<1.5$} & \multicolumn{3}{|c|}{$1.5<z<2.3$} \\
\hline & $\begin{array}{c}\text { Number } \\
\text { of Sources }\end{array}$ & $\begin{array}{c}\text { Density } \\
\left(\mathrm{Mpc}^{-3} d \log L^{-1}\right)\end{array}$ & $\begin{array}{l}\text { Corrected Density } \\
\left(\mathrm{Mpc}^{-3} d \log L^{-1}\right)\end{array}$ & $\begin{array}{l}\text { Number } \\
\text { of Sources }\end{array}$ & $\begin{array}{c}\text { Density } \\
\left(\mathrm{Mpc}^{-3} d \log L^{-1}\right)\end{array}$ & $\begin{array}{l}\text { Corrected Density } \\
\left(\mathrm{Mpc}^{-3} d \log L^{-1}\right)\end{array}$ \\
\hline 41.45 & 36 & 0.00097 & $0.0016 \pm 0.00034$ & $\mathrm{n} / \mathrm{a}$ & $\mathrm{n} / \mathrm{a}$ & $\mathrm{n} / \mathrm{a}$ \\
\hline 41.75 & 68 & 0.0013 & $0.0020 \pm 0.00032$ & $\mathrm{n} / \mathrm{a}$ & $\mathrm{n} / \mathrm{a}$ & $\mathrm{n} / \mathrm{a}$ \\
\hline 42.05 & 55 & 0.00085 & $0.0012 \pm 0.00020$ & 11 & 0.00016 & $0.00059 \pm 0.00020$ \\
\hline 42.35 & 25 & 0.00038 & $0.00055 \pm 0.00012$ & 20 & 0.00022 & $0.00077 \pm 0.00021$ \\
\hline 42.65 & 7 & 0.00011 & $0.00013 \pm 5.3 \mathrm{e}-05$ & 16 & 0.00017 & $0.00036 \pm 0.00010$ \\
\hline 42.95 & 1 & $1.5 \mathrm{e}-05$ & $1.8 \mathrm{e}-05 \pm 1.8 \mathrm{e}-05$ & 7 & $7.0 \mathrm{e}-05$ & $0.00016 \pm 6.5 \mathrm{e}-05$ \\
\hline 43.25 & $\mathrm{n} / \mathrm{a}$ & $\mathrm{n} / \mathrm{a}$ & $\mathrm{n} / \mathrm{a}$ & 2 & $2.0 \mathrm{e}-05$ & $2.2 \mathrm{e}-05 \pm 1.6 \mathrm{e}-05$ \\
\hline 43.55 & $\mathrm{n} / \mathrm{a}$ & $\mathrm{n} / \mathrm{a}$ & $\mathrm{n} / \mathrm{a}$ & 2 & $1.9 \mathrm{e}-05$ & $2.2 \mathrm{e}-05 \pm 1.6 \mathrm{e}-05$ \\
\hline
\end{tabular}

Notes.

a No extinction correction has been applied.

${ }^{\mathrm{b}}$ Corrected density is the measured number density with completeness corrections applied.

However, our measured luminosity bins do not constrain $\alpha$ well. If we require the $0.7<z<1.5 \alpha$ to be the same as seen at low redshift $(\alpha=-1.5)$, we find only minor changes in $\Phi_{\star}$, with all the evolution in [O III] from $z=0.7-2.3$ taking place in $L_{\star}$.

\section{SUMMARY AND CONCLUSIONS}

We present near-infrared emission line number densities, line ratios, and luminosity functions, based on 29 fields from the WISP survey taken with both the G102+G141 grism filters. With this data set we find emission-line galaxies without any pre-selection bias, over a large continuous epoch of cosmic time. At least two future space missions, Euclid and WFIRST, will also contain very large near-infrared grism surveys. The WISP survey is a perfect laboratory to predict what these future missions can expect to find.

We found the most significant potential issues with identifying emission lines from near-infrared grism spectroscopy to be:

1. Confusion of $\mathrm{H \alpha}$ and [O III] due to insufficient wavelength resolution, which can lead to catastrophic redshift identification failures. When the $\mathrm{S} / \mathrm{N}$ gets low enough, one cannot necessarily depend on being able to distinguish the [O III] doublet from a single emission line. This is particularly true in the case where the emission-line region is spatially extended, which effectively degrades the wavelength resolution. This could potentially be a significant issue for shallower surveys, as the brighter emission line sources tend to also be larger in spatial extent. However, we found this line confusion to be almost entirely confined to the lower resolution $(R \sim 130)$ G141 grism and not an issue for the higher resolution $(R \sim 210)$ G102 grism. Additionally, our simulations found that the higher grism resolution is also needed to achieve the $0.1 \%$ accuracy in $1+z$ required for $\mathrm{BAO}$ experiments. We would therefore strongly recommend that future near-IR missions keep to wavelength resolutions above $R>200$.

2. Contamination from overlapping spectra from bright sources, which effects even bright, high $\mathrm{S} / \mathrm{N}$ spectra. It alters the measured continuum, impacts the effectiveness of automatic line-finding algorithms, makes it difficult to identify the correct host galaxy and emissionline wavelength, and results in emission lines that are lost altogether. Roughly $5 \%$ of all our bright lines $(>3 \times$ $10^{-16} \mathrm{erg} \mathrm{s}^{-1} \mathrm{~cm}^{-2}$ ) are lost to overlapping bright source contamination. The use of multiple roll angles would greatly minimize these effects, particularly for the brighter, less densely packed sources mostly being targeted by future missions.

3. Lack of dithering, which not only greatly impacts our ability to mitigate cosmic rays, hot and warm pixels, and other artifacts that can mimic emission lines, but also prevents us from recovering the additional resolution that sub-pixel dithering can provide. With grism spectroscopy, this results in not only improved spatial resolution but also improved wavelength resolution, which helps to address the insufficient wavelength resolution issue already discussed above.

While WISP is sensitive to $\mathrm{H} \alpha$ emitters down to $z=0.3$, both Euclid and WFIRST will likely have low-wavelength cut-offs around $1.1 \mu \mathrm{m}$, making them mostly sensitive to emission-line galaxies at $z>0.7$. We find that our cumulative number of $\mathrm{H} \alpha$ galaxies at $0.7<z<1.5$ reaches $10,000 \mathrm{deg}^{-2}$ by an $\mathrm{H} \alpha$ flux of $2 \times 10^{-16} \mathrm{erg} \mathrm{s}^{-1} \mathrm{~cm}^{-2}$. H $\alpha$-emitting galaxies with comparable [O III] flux are roughly five times less common at these $(\sim 1-3$ $\times 10^{-16} \mathrm{erg} \mathrm{s}^{-1} \mathrm{~cm}^{-2}$ ) emission-line fluxes.

As emission-line fluxes become fainter, the $\mathrm{H} \alpha /[\mathrm{O}$ III $]$ ratio becomes smaller, largely a result of the correlation between the $\mathrm{H} \alpha /[\mathrm{O} \mathrm{III}]$ ratio and $\mathrm{H} \alpha$ luminosity previously reported by WISP (Domínguez et al. 2013). While great numbers of $\mathrm{H} \alpha /\left[\mathrm{O}_{\mathrm{III}}\right]<1$ emission-line galaxies can be found around $5 \times 10^{-17} \mathrm{erg} \mathrm{s}^{-1} \mathrm{~cm}^{-2}$, there are effectively none in our sample once the $\mathrm{H} \alpha$ flux becomes greater than $2 \times 10^{-16} \mathrm{erg} \mathrm{s}^{-1} \mathrm{~cm}^{-2}$. These large $\mathrm{H} \alpha /[\mathrm{O} \mathrm{III}]$ ratios for the brighter emission lines suggest that $60 \%-80 \%$ of the $\mathrm{H} \alpha$ emission lines found by future space missions will be single lines. However, that same high $\mathrm{H} \alpha /[\mathrm{O} \mathrm{III}]$ ratio suggests that the likelihood of contamination by lines besides $\mathrm{H} \alpha$ remains low.

Our $\mathrm{H} \alpha$ luminosity function finds a similar number density of line emitters to that of the NICMOS near-infrared grism surveys at faint luminosities, but finds significantly fewer numbers of line emitters (factors of 3-4 less) at the bright luminosity end. We find our $0.9<z<1.5$ number counts to be consistent with the $z=1.47$ narrowband $\mathrm{H} \alpha$ survey of HiZELS (Sobral et al. 2013), although with our lower median redshift $z=1.15$, we would have expected slightly lower densities if the luminosity function is smoothly evolving out to $z=1.5$. On the other hand, our lower redshift $0.3<z<0.9 \mathrm{H} \alpha$-emitter number counts fall just below both the narrowband counts from HiZELS at $z=0.84$ and NEWFIRM $\mathrm{H} \alpha$ at $z=0.8$. The absolute difference 
in expected luminosity density for the higher redshifts is small and could be partially a result of cosmological variance of the narrowband sample.

The [O III] emission line probes a higher redshift range than $\mathrm{H} \alpha$ and is therefore not sensitive to the fainter luminosities required to properly constrain $\alpha$. If we fix $\alpha=-1.5$, as seen in studies at lower redshifts, we find that the evolution in the [O III] luminosity function from $z=0.7-2.3$ is almost entirely in the $L_{\star}$ parameter, similar to what we observe for the $z=0.3-1.5$ evolution of the $\mathrm{H} \alpha$ luminosity function.

We would like to acknowledge the assistance of Chun Ly for his helpful advice during the production of this paper. The authors would also like to acknowledge financial support from the grants for HST programs GO-10226, GO-11696, and GO-12283.

\section{REFERENCES}

Atek, H., Malkan, M., McCarthy, P., et al. 2010, ApJ, 723, 104

Atek, H., Siana, B., Scarlata, C., et al. 2011, ApJ, 743, 121

Brammer, G. B., van Dokkum, P. G., Franx, M., et al. 2012, ApJS, 200, 13

Cole, S., Percival, W. J., Peacock, J. A., et al. 2005, MNRAS, 362, 505

Domínguez, A., Siana, B., Henry, A. L., et al. 2013, ApJ, 763, 145

Dressler, A., Spergel, D., Mountain, M., et al. 2012, arXiv:1210.7809

Eisenstein, D. J., \& Hu, W. 1998, ApJ, 496, 605

Eisenstein, D. J., Zehavi, I., Hogg, D. W., et al. 2005, ApJ, 633, 560

Felten, J. E. 1977, AJ, 82, 861

Fumagalli, M., Patel, S. G., Franx, M., et al. 2012, ApJL, 757, L22

Garn, T., Sobral, D., Best, P. N., et al. 2010, MNRAS, 402, 2017

Geach, J. E., Cimatti, A., Percival, W., et al. 2010, MNRAS, 402, 1330

Geach, J. E., Smail, I., Best, P. N., et al. 2008, MNRAS, 388, 1473

Glazebrook, K., Baldry, I., Moos, W., Kruk, J., \& McCandliss, S. 2005, NewAR, 49, 374

Green, J., Schechter, P., Baltay, C., et al. 2012, arXiv:1208.4012

Henry, A. L., Martin, C. L., Dressler, A., Sawicki, M., \& McCarthy, P. 2012, ApJ, 744,149

Hopkins, A. M., Connolly, A. J., \& Szalay, A. S. 2000, AJ, 120, 2843
Hu, E. M., Cowie, L. L., Kakazu, Y., \& Barger, A. J. 2009, ApJ, 698, 2014

Ilbert, O., McCracken, H. J., Le Fèvre, O., et al. 2013, A\&A, 556, A55

Kimble, R. A., MacKenty, J. W., O’Connell, R. W., \& Townsend, J. A. 2008, Proc. SPIE, 7010, 43

Kobulnicky, H. A., \& Kewley, L. J. 2004, ApJ, 617, 240

Kümmel, M., Kuntschner, H., \& Walsh, J. 2007, STECF, 43, 8

Kümmel, M., Walsh, J. R., Pirzkal, N., Kuntschner, H., \& Pasquali, A. 2009, PASP, 121, 59

Laureijs, R., Amiaux, J., Arduini, S., et al. 2011, arXiv:1110.3193

Laureijs, R., Gondoin, P., Duvet, L., et al. 2012, Proc. SPIE, 8442, 84420T

Lee, J. C., Kennicutt, R. C., Funes, S. J., et al. 2007, ApJL, 671, L113

Lee, J. C., Ly, C., Spitler, L., et al. 2012, PASP, 124, 782

Liang, Y. C., Yin, S. Y., Hammer, F., et al. 2006, ApJ, 652, 257

Ly, C., Lee, J. C., Dale, D. A., et al. 2011, ApJ, 726, 109

Ly, C., Malkan, M. A., Kashikawa, N., et al. 2007, ApJ, 657, 738

MacKenty, J. W., Kimble, R. A., O’Connell, R. W., \& Townsend, J. A. 2010, Proc. SPIE, 7731, 27

Magnelli, B., Elbaz, D., Chary, R. R., et al. 2011, A\&A, 528, A35

Martin, C. L., Sawicki, M., Dressler, A., \& McCarthy, P. 2008, ApJ, 679, 942

McCarthy, P. J., Yan, L., Freudling, W., et al. 1999, ApJ, 520, 548

McLure, R. J., Jarvis, M. J., Targett, T. A., Dunlop, J. S., \& Best, P. N. 2006, MNRAS, 368, 1395

Muzzin, A., Marchesini, D., Stefanon, M., et al. 2013, ApJ, 777, 18

Osterman, S., Green, J., Froning, C., et al. 2011, Ap\&SS, 335, 257

Pérez-González, P. G., Rieke, G. H., Villar, V., et al. 2008, ApJ, 675, 234

Pirzkal, N., Rothberg, B., Ly, C., et al. 2013, ApJ, 772, 48

Shim, H., Colbert, J., Teplitz, H., et al. 2009, ApJ, 696, 785

Sobral, D., Best, P. N., Geach, J. E., et al. 2009, MNRAS, 398, 75

Sobral, D., Best, P. N., Matsuda, Y., et al. 2012, MNRAS, 420, 1926

Sobral, D., Smail, I., Best, P. N., et al. 2013, MNRAS, 428, 1128

Thompson, R. I., \& Schneider, G. 1998, Proc. SPIE, 3356, 215

Tremonti, C. A., Heckman, T. M., Kauffmann, G., et al. 2004, ApJ, 613, 898

Trenti, M., \& Stiavelli, M. 2008, ApJ, 676, 767

van der Wel, A., Straughn, A. N., Rix, H.-W., et al. 2011, ApJ, 742, 111

van Dokkum, P. G., Leja, J., Nelson, E. J., et al. 2013, ApJL, 771, L35

van Dokkum, P. G., Whitaker, K. E., Brammer, G., et al. 2010, ApJ, 709, 1018

Wang, Y., Chuang, C.-H., \& Hirata, C. M. 2013, MNRAS, 430, 2446

Wang, Y., Percival, W., Cimatti, A., et al. 2010, MNRAS, 409, 737

Weinberg, D. H., Mortonson, M. J., Eisenstein, D. J., et al. 2012, PhR, 530, 87 Weiner, B. J. 2012, arXiv:1209.1405

Woodgate, B. E., Kimble, R. A., Bowers, C. W., et al. 1998, PASP, 110, 1183

Yan, L., McCarthy, P. J., Freudling, W., et al. 1999, ApJL, 519, L47 